\title{
Autocorrelation of ratios of $L$-functions
}

\author{
Brian Conrey, David W. Farmer and Martin R. Zirnbauer
}

\begin{abstract}
We give a new heuristic for all of the main terms in the quotient of products of $L$-functions averaged over a family. These conjectures generalize the recent conjectures for mean values of $L$-functions. Comparison is made to the analogous quantities for the characteristic polynomials of matrices averaged over a classical compact group.
\end{abstract}

\section{Introduction}

Conjectures for the moments of $L$-functions have been sought for many decades, with very little progress until the introduction of random matrix theory into the subject $[4,26-28]$. The predictions using random matrix theory provide plausible conjectures for the leading order asymptotics of the moments of a family of $L$-functions. More recently, conjectures for all of the main terms of the moments have been found, using heuristics based on the harmonic detector of a family [6] and also using a plausible conjecture for multiple Dirichlet series [13]. Although the more refined conjectures do not make use of random matrix theory, they are supported by the similarity to the analogous theorems for random matrices [5].

In this paper, we generalize the heuristic method of [6] to the case of ratios of products of $L$-functions. In our companion paper [8] and in [21], the methods of supersymmetry are used to evaluate the analogous quantity for the characteristic polynomials of matrices averaged over the compact classical groups. We quote those results in Section 4 for comparison with our conjectures given in Section 5 .

The usefulness of averages of ratios was first suggested by Farmer [14], who made the following First Ratios Conjecture about the Riemann zetafunction. For complex numbers $\alpha, \beta, \gamma, \delta$ with real parts that are positive and of size $c / \log T$,

$$
\frac{1}{T} \int_{0}^{T} \frac{\zeta(s+\alpha) \zeta(1-s+\beta)}{\zeta(s+\gamma) \zeta(1-s+\delta)} d t \sim 1+\left(1-T^{-\alpha-\beta}\right) \frac{(\alpha-\gamma)(\beta-\delta)}{(\alpha+\beta)(\gamma+\delta)}
$$


This conjecture, developed in conjunction with the conjecture on "long mollifiers," had a somewhat shaky justification, yet it implied many things of great interest about the Riemann zeta-function, such as the pair correlation conjecture of Montgomery [30], Levinson's formula for a "mollified" mean square of $\zeta(s)$ and an asymptotic formula for moments of the logarithmic derivative of the zeta-function near the critical line. Moreover, it satisfied many consistency checks involving exchanging parameters, using the functional equation of $\zeta(s)$ and letting variables approach 0 or $\infty$. Farmer later extended the conjecture to include similar integrals but with ratios of up to three zeta-functions in the numerator and denominator [15]. These formulas were also found to imply interesting statements about the zeros of $\zeta(s)$, including the triple correlation conjecture of Hejhal [20] and Rudnick-Sarnak [31].

In another direction, Goldston and Montgomery [19] have shown that the pair correlation conjecture is equivalent to a statement about the second moment of primes in short intervals. In [18], an asymptotic formula for the mean-square of the logarithmic derivative of $\zeta(s)$ was shown to be equivalent to both the pair correlation and (hence) the second moment of primes. Conjecture (1.1) encapsulates these results.

Thus, it was of great interest that the analog of formula (1.1) was found to be true for the characteristic polynomial of a matrix averaged over the unitary group $U(N)$. This was first observed by Nonenmacher and Zirnbauer at a workshop at MSRI in 1999. Since it is believed that families of $L$-functions can be modeled by the characteristic polynomials from one of the classical compact groups, these formulas for other compact groups lead to conjectures for the averages, over a family, of ratios of products of $L$-functions. In every case where we can prove an asymptotic formula, or conjecture one from number theoretic reasoning, we have agreement with the conjectures presented here. We noted above that the ratios conjectures imply Montgomery's pair correlation conjecture. The ratios conjectures contain much more information and can be used to make very precise conjectures about the distribution of zeros of $L$-functions. In addition to the examples we give in Section 7 of this paper, Conrey and Snaith [10] have given a large number of applications.

In Section 2, we give an outline of some basic properties of matrix groups; then in Section 3 we give some examples of families of $L$-functions. In Section 4, we present the theorems for ratios of characteristic polynomials, quoted from $[8,21]$. In Section 5, we describe our method of conjecturing precise formulas for averages of ratios (an elaboration of the recipe in [6]), and we give several examples. Refinements of the conjectures are presented in Section 6. Finally, we mention some applications in Section 7. 


\section{Random matrices and $L$-functions}

We review the properties of the characteristic polynomials of classical matrices which we consider in this paper.

\subsection{Unitary matrices}

If $A=\left(a_{j k}\right)$ is an $N \times N$ matrix with complex entries, we let $A^{*}$ be its conjugate transpose, i.e., $A^{*}=\left(b_{j k}\right)$ where $b_{j k}=\overline{a_{k j}}$. $A$ is said to be unitary if $A A^{*}=I$. We let $U(N)$ denote the group of all $N \times N$ unitary matrices. This is a compact Lie group and has a Haar measure which allows us to do analysis.

All of the eigenvalues of $A \in U(N)$ have absolute value 1 ; we write them as

$$
\mathrm{e}^{i \theta_{1}}, \mathrm{e}^{i \theta_{2}}, \ldots, \mathrm{e}^{i \theta_{N}}
$$

with

$$
0 \leq \theta_{j}<2 \pi
$$

The eigenvalues of $A^{*}$ are $\mathrm{e}^{-i \theta_{1}}, \ldots, \mathrm{e}^{-i \theta_{N}}$. The determinant, $\operatorname{det} A=$ $\prod_{n=1}^{N} \mathrm{e}^{i \theta_{n}}$, is a complex number with absolute value 1 .

For any sequence of $N$ points on the unit circle there are matrices in $U(N)$ with those points as eigenvalues. The collection of all matrices with the same set of eigenvalues constitutes a conjugacy class in $U(N)$. Thus, the set of conjugacy classes can identified with the collection of sets of $N$ points on the unit circle.

The characteristic polynomial of the matrix $A$ is denoted $\Lambda_{A}(s)$ and is defined by

$$
\Lambda_{A}(s)=\operatorname{det}\left(I-s A^{*}\right)=\prod_{n=1}^{N}\left(1-s \mathrm{e}^{-i \theta_{n}}\right)
$$

The roots of $\Lambda_{A}(s)$ are the eigenvalues of $A$ and are on the unit circle. The characteristic polynomial satisfies the functional equation

$$
\begin{aligned}
\Lambda_{A}(s) & =(-s)^{N} \prod_{n=1}^{N} \mathrm{e}^{-i \theta_{n}} \prod_{n=1}^{N}\left(1-\mathrm{e}^{i \theta_{n}} / s\right) \\
& =(-1)^{N} \operatorname{det} A^{*} s^{N} \Lambda_{A^{*}}(1 / s) .
\end{aligned}
$$


We regard $\Lambda_{A}(s)$ as an analog of the Riemann zeta-function, where the eigenangle $\theta$ plays the role of the parameter $t$ on the critical line.

\subsection{Symplectic matrices}

The unitary matrix $A$ is said to be symplectic if $A Z A^{t}=Z$, where

$$
Z=\left(\begin{array}{cc}
0 & I_{N} \\
-I_{N} & 0
\end{array}\right)
$$

where $A^{t}$ denotes the transpose of $A$. A symplectic matrix necessarily has determinant 1 . The symplectic group $U \operatorname{Sp}(2 N)$ is the group of $2 N \times 2 N$ unitary symplectic matrices. The eigenvalues of a symplectic matrix occur in complex conjugate pairs and we write them as

$$
\mathrm{e}^{ \pm i \theta_{1}}, \ldots, \mathrm{e}^{ \pm i \theta_{N}}
$$

with

$$
0 \leq \theta_{j} \leq \pi
$$

The functional equation of the characteristic polynomial is

$$
\Lambda_{A}(s)=s^{2 N} \Lambda_{A^{t}}(1 / s)
$$

\subsection{Orthogonal matrices}

A unitary matrix $A$ is said to be orthogonal if $A A^{t}=I$. Orthogonality for a unitary matrix implies that $A^{t}=A^{*}$ or $\bar{A}=A$. In other words, an orthogonal matrix is a real unitary matrix. We let $\mathrm{SO}(N)$ denote the subgroup of $U(N)$ consisting of $N \times N$ orthogonal matrices with determinant 1 .

The functional equation of the characteristic polynomial is

$$
\Lambda_{A}(s)=(-1)^{N} s^{N} \Lambda_{A^{t}}(1 / s)
$$

Thus, if $N$ is even, then the sign in the functional equation is 1 and if $N$ is odd then the sign is -1 . We distinguish these two cases as "even" orthogonal and "odd" orthogonal. 
For any complex eigenvalue of an orthogonal matrix, its complex conjugate is also an eigenvalue. The eigenvalues of $A \in \mathrm{SO}(2 N)$ can be written as

$$
\mathrm{e}^{ \pm i \theta_{1}}, \ldots, \mathrm{e}^{ \pm i \theta_{N}}
$$

and the eigenvalues of $A \in \mathrm{SO}(2 N+1)$ can be written as

$$
1, \mathrm{e}^{ \pm i \theta_{1}}, \ldots, \mathrm{e}^{ \pm i \theta_{N}}
$$

where in both cases

$$
0 \leq \theta_{j} \leq \pi
$$

\section{3. $L$-functions}

We briefly describe some of the families of $L$-functions for which we can formulate a ratios conjecture; see [16] for an introduction and [6] for all the details. Much of the present paper is an extension of [6] and we assume some familiarity with that paper.

For the purpose of making conjectures for ratios, the main feature of a family is that it is partially ordered by a parameter (usually called the "conductor"), and there is a "harmonic detector" which describes the behaviour of the coefficients when averaged over the family.

\subsection{The Riemann zeta-function}

The Riemann zeta-function [32] is defined by

$$
\zeta(s)=\sum_{n=1}^{\infty} \frac{1}{n^{s}}
$$

for $s=\sigma+i t$ with $\sigma>1$. It has a meromorphic continuation to the whole complex plane with its only singularity a simple pole at $s=1$ with residue 1 . It satisfies a functional equation which in its symmetric form reads

$$
\pi^{-s / 2} \Gamma\left(\frac{s}{2}\right) \zeta(s)=\pi^{(s-1) / 2} \Gamma\left(\frac{1-s}{2}\right) \zeta(1-s)
$$

and in its asymmetric form

$$
\zeta(s)=\chi(s) \zeta(1-s)
$$


where

$$
\chi(1-s)=\chi(s)^{-1}=2(2 \pi)^{-s} \Gamma(s) \cos \frac{\pi s}{2} .
$$

The product formula discovered by Euler is

$$
\zeta(s)=\prod_{p}\left(1-\frac{1}{p^{s}}\right)^{-1}
$$

for $\sigma>1$ where the product is over the prime numbers $p$.

The family $\{\zeta(1 / 2+i t) \mid t>0\}$ parameterized by real numbers $t$ can be modeled by characteristic polynomials of unitary matrices. We will use a modification of the recipe in [6] to conjecture mean values for ratios of products of $\zeta$-functions. The key ingredient in the recipe is the orthogonality relation (or "harmonic detector")

$$
\lim _{T \rightarrow \infty} \frac{1}{T} \int_{0}^{T}\left(\frac{m}{n}\right)^{i t} d t= \begin{cases}1 & \text { if } m=n \\ 0 & \text { if } m \neq n\end{cases}
$$

\subsection{Dirichlet $L$-functions with real characters}

We let

$$
L\left(s, \chi_{d}\right)=\sum_{n=1}^{\infty} \frac{\chi_{d}(n)}{n^{s}}
$$

for $\Re s>1$ where $\chi_{d}(n)$ is a primitive, real Dirichlet character [11]. The complete set of these characters is described below. Each of these (with $|d|>$ 1 ) is an entire function of $s$ and, if $d>0$, satisfies the functional equation

$$
\left(\frac{\pi}{|d|}\right)^{-s / 2} \Gamma\left(\frac{s}{2}\right) L\left(s, \chi_{d}\right)=\left(\frac{\pi}{|d|}\right)^{(s-1) / 2} \Gamma\left(\frac{1-s}{2}\right) L\left(1-s, \chi_{d}\right)
$$

whereas if $d<0$, satisfies the functional equation

$$
\left(\frac{\pi}{|d|}\right)^{-s / 2} \Gamma\left(\frac{s+1}{2}\right) L\left(s, \chi_{d}\right)=\left(\frac{\pi}{|d|}\right)^{(s-1) / 2} \Gamma\left(\frac{2-s}{2}\right) L\left(1-s, \chi_{d}\right) .
$$

We now describe the characters $\chi_{d}$. These are not defined for all $d$ but only for $d$ which are known as fundamental discriminants. The values taken 
on by $\chi_{d}(n)$ are $0,-1$ and +1 . We begin with $\chi_{-4}(n)$ which is defined to be 1 if $n \equiv 1 \bmod 4$, is defined to be -1 if $n \equiv 3 \bmod 4$, and is 0 if $n$ is even. Next, we have $\chi_{-8}(n)$ which is defined to be 0 if $n$ is even, is +1 if $n \equiv 1$ or $3 \bmod 8$ and is -1 if $n \equiv 5$ or $7 \bmod 8$. We also have $\chi_{8}(n)$ which is defined to be 0 if $n$ is even, is +1 if $n \equiv 1$ or $7 \bmod 8$ and is -1 if $n \equiv 3$ or $5 \bmod 8$. This takes care of all of the $d$ which are plus or minus a power of 2 . Now $d$ can also be equal to a prime $p \equiv 1 \bmod 4$. In this case $\chi_{p}(n)$ is 0 if $n$ is divisible by $p$, is +1 if $n \equiv a^{2} \bmod p$ for some $a$ not divisible by $p$ and is -1 otherwise. If $p \equiv 3 \bmod 4$, then there is a character $\chi_{-p}(n)$ which is defined exactly as $\chi_{p}(n)$ for a $p \equiv 1 \bmod 4$. Finally, we can take any pointwise product of distinct $\chi_{d}(n)$ to form

$$
\chi_{d_{1} \cdot d_{2} \cdots \cdots d_{u}}(n)=\chi_{d_{1}}(n) \chi_{d_{2}}(n) \cdots \chi_{d_{u}}(n) .
$$

If we take the empty product, then we have the character $\chi_{1}(n)=1$ for all $n$, so that $L\left(s, \chi_{1}\right)=\zeta(s)$. This completes the description of all of the primitive real characters.

Note that each $\chi_{d}(n)$ is defined for all integers $n$, positive and negative. Also, $\chi_{d}(n)$ is periodic in $n$ with smallest period equal to $|d|$. These functions are completely multiplicative, which means that

$$
\chi_{d}(m n)=\chi_{d}(m) \chi_{d}(n) .
$$

This multiplicativity implies that $L\left(s, \chi_{d}\right)$ has an Euler product formula

$$
L\left(s, \chi_{d}\right)=\prod_{p}\left(1-\frac{\chi_{d}(p)}{p^{s}}\right)^{-1}
$$

valid for $\Re s>1$. If $d<0$, then $\chi_{d}$ is odd (i.e., $\chi_{d}(-n)=-\chi_{d}(n)$ ), whereas if $d>0$, then $\chi_{d}(n)$ is even. We saw above that the functional equations are slightly different in the even and odd cases.

The collection of fundamental discriminants can be described as the set of $d$ which either are square-free and congruent to 1 modulo 4 or are four times a square-free number which is congruent to 2 or 3 modulo 4 . The sequence of $d$ is

$$
\ldots,-24,-23,-20,-19,-15,-11,-8,-7,-4,-3,1,5,8,12,
$$

$$
13,17,21,24,28,29,33,37, \ldots \text {. }
$$

The families $\left\{L\left(1 / 2, \chi_{d}\right) \mid d<0\right\}$ and $\left\{L\left(1 / 2, \chi_{d}\right) \mid d>0\right\}$, parameterized, respectively, by positive and negative fundamental discriminants, can each 
be modeled by characteristic polynomials of symplectic matrices. The harmonic detector for the positive discriminant family is

$$
\begin{aligned}
\delta(n) & :=\lim _{X \rightarrow \infty} \frac{1}{X^{*}} \sum_{0<d \leq X} \chi_{d}(n) \\
& = \begin{cases}\prod_{p \mid n}(1+1 / p)^{-1} & \text { if } n \text { is a perfect square, } \\
0 & \text { if } n \text { is not a perfect square, }\end{cases}
\end{aligned}
$$

where $X^{*}$ is the number of fundamental discriminants $0<d \leq X$. A similar formula holds for a sum over the odd characters $0<-d<X$.

\subsection{Quadratic twists of a modular $L$-function}

We now give an example of an orthogonal family of $L$-functions.

Let $f(z)$ be a holomorphic newform with integer coefficients [25]. For example, if

$$
F(q)=q \prod_{n=1}^{\infty}\left(1-q^{n}\right)^{2}\left(1-q^{11 n}\right)^{2}=\sum_{n=1}^{\infty} a_{n} q^{n}
$$

where $a_{1}=1, a_{2}=-2, a_{3}=-1, a_{4}=2, \ldots$, then

$$
f(z)=F\left(\mathrm{e}^{2 \pi i z}\right)=\sum_{n=1}^{\infty} a_{n} \mathrm{e}^{2 \pi i n z}
$$

is a newform (i.e., it is an eigenfunction of the appropriate Hecke operators). Specifically, $f(z)$ is a cusp form of weight 2 for

$$
\Gamma_{0}(11)=\left\{\left(\begin{array}{ll}
a & b \\
c & d
\end{array}\right) \in S L(2, \mathbb{Z}): 11 \mid c\right\}
$$

that is

$$
f\left(\frac{a z+b}{c z+d}\right)=(c z+d)^{2} f(z) \text { for all }\left(\begin{array}{ll}
a & b \\
c & d
\end{array}\right) \in \Gamma_{0}(11)
$$

The exponent of the factor multiplying $f(z)$ is the weight, which is 2 in this case. 
This newform is associated with an elliptic curve. Denote by $E_{11}$ the elliptic curve

$$
y^{2}=4 x^{3}-4 x^{2}-40 x-79,
$$

and let

$$
N_{p}=\#\left\{(x, y): y^{2} \equiv 4 x^{3}-4 x^{2}-40 x-79 \bmod p\right\},
$$

then the coefficients satisfy

$$
a_{p}=p-N_{p}
$$

Deligne's theorem (proved earlier by Hasse for the special case of elliptic curves) states that for a weight- $k$ newform we have $\left|a_{p}\right|<2 p^{(k-1) / 2}$, and so in our example $\left|a_{p}\right|<2 \sqrt{p}$. We write

$$
\lambda_{E}(n)=\lambda(n)=\frac{a(n)}{\sqrt{n}} .
$$

Therefore, one may associate with a newform $f$ a Dirichlet series, called the $L$-function of the modular form,

$$
L_{E_{11}}(s)=\sum_{n=1}^{\infty} \frac{\lambda(n)}{n^{s}}
$$

which converges absolutely for $\Re s>1$. The coefficients $\lambda(n)$ also satisfy the Hecke relations

$$
\lambda(m) \lambda(n)=\sum_{\substack{d|m \\ d| n}} \lambda\left(\frac{m n}{d^{2}}\right)
$$

which implies that the $L$-function has an Euler product

$$
L_{E_{11}}(s)=\left(1-\frac{1}{11^{s+1 / 2}}\right)^{-1} \prod_{p \neq 11}\left(1-\frac{\lambda(p)}{p^{s}}+\frac{1}{p^{2 s}}\right)^{-1} .
$$

The $L$-function associated with an elliptic curve is entire and satisfies the functional equation

$$
\left(\frac{\sqrt{M}}{2 \pi}\right)^{s} \Gamma(s) L_{E}(s)=w(E)\left(\frac{\sqrt{M}}{2 \pi}\right)^{1-s} \Gamma(1-s) L_{E}(1-s)
$$

where $M$ is the conductor of the elliptic curve $E$ and $w(E)= \pm 1$ is called the sign of the functional equation. For $E_{11}$, we have $M=11$ and $w(E)=1$. 
The family we want to describe is the collection of $L$-functions associated with the quadratic twists of a fixed $L$-function. Let $L_{E}$ be the $L$-function associated with an elliptic curve $E$ and let $\chi_{d}(n)$ be a real primitive Dirichlet character, as described in the previous section. Then the twisted $L$-function

$$
L_{E}\left(s, \chi_{d}\right)=\sum_{n=1}^{\infty} \frac{\lambda(n) \chi_{d}(n)}{n^{s}}
$$

is the $L$-function of another elliptic curve $E_{d}$ : the quadratic twist of $E$ by $d$. It can be shown that the number $N_{p, d}$ of solutions of $E_{d}$ modulo $p$ satisfies

$$
p-N_{p, d}=\chi_{d}(p) a_{p}
$$

Moreover, $L_{E_{d}}(s)=L_{E}\left(s, \chi_{d}\right)$ satisfies the functional equation

$$
\left(\frac{\sqrt{M}|d|}{2 \pi}\right)^{s} \Gamma(s) L_{E_{d}}(s)=\chi_{d}(-M) w(E)\left(\frac{\sqrt{M}|d|}{2 \pi}\right)^{1-s} \Gamma(1-s) L_{E_{d}}(1-s)
$$

For example, the quadratic twist of $E_{11}$ by $d$ is the elliptic curve

$$
d y^{2}=4 x^{3}-4 x^{2}-40 x-79
$$

The corresponding twisted $L$-function is

$$
\begin{aligned}
\left.L_{E_{11}}\left(s, \chi_{d}\right)\right) & =\sum_{n=1}^{\infty}\left(\frac{d}{n}\right) \frac{\lambda(n)}{n^{s}} \\
& =\left(1-\left(\frac{d}{11}\right) \frac{1}{11^{s+1 / 2}}\right)^{-1} \prod_{p \nmid 11 d}\left(1-\left(\frac{d}{n}\right) \frac{\lambda(p)}{p^{s}}+\frac{1}{p^{2 s}}\right)^{-1} .
\end{aligned}
$$

This satisfies the functional equation

$$
\left(\frac{\sqrt{11}|d|}{2 \pi}\right)^{s} \Gamma(s) L_{E_{11, d}}(s)=\chi_{d}(-11)\left(\frac{\sqrt{11}|d|}{2 \pi}\right)^{1-s} \Gamma(1-s) L_{E_{11, d}}(1-s) .
$$


Note that when $d \equiv 2,6,7,8$, or $10 \bmod 11$, then the sign in the functional equation is +1 . The $L_{E_{11}}\left(s, \chi_{d}\right)$ for these $d$ form an even orthogonal family. If $d \equiv 1,3,4,5$ or $9 \bmod 11$, then the sign in the functional equation is -1 and the $L_{E_{11}}\left(s, \chi_{d}\right)$ for these $d$ form an odd orthogonal family.

\section{Autocorrelation of ratios of characteristic polynomials}

We quote formulas from $[8,21]$ for the ratios of characteristic polynomials averaged over the unitary, symplectic and orthogonal matrix groups. Variants of these formulas have also been given by Basor and Forrester [2], Day [12], Baik et al. [1], Fyodorov and Strahov [17] and others. New proofs for these formulas, in restricted ranges, have also recently been given by Conrey et al. [7] and Bump and Gamburd [3].

Note that in the case of an equal number of characteristic polynomials in the numerator and denominator, the results we quote from $[8,21]$ are valid for all $N$, while the other methods are only valid for sufficiently large $N$.

We let

$$
z(x)=\frac{1}{1-\mathrm{e}^{-x}}=\frac{1}{x}+O(1)
$$

It will be seen that the function $z(x)$ plays the role for random matrix theory that $\zeta(1+x)$ plays in the theory of moments of $L$-functions.

Also let $\Xi_{K, L}$ denote the subset of permutations $\sigma \in S_{K+L}$ of $\{1,2, \ldots$, $K+L\}$ for which

$$
\sigma(1)<\sigma(2)<\cdots<\sigma(K)
$$

and

$$
\sigma(K+1)<\sigma(K+2)<\cdots<\sigma(K+L) .
$$

The cardinality of $\Xi_{K, L}$ is $\left(\begin{array}{c}K+L \\ K\end{array}\right)=\frac{(K+L) !}{K ! L !}$. Finally, let $\epsilon=\left(\epsilon_{1}, \ldots, \epsilon_{K}\right)$ be a vector with each component $\epsilon_{k}= \pm 1$ and denote $\operatorname{sgn}(\epsilon)=\prod_{k=1}^{K} \epsilon_{k}$.

We let $d A$ denote the Haar measure on whichever group we are integrating over. 
Theorem 4.1. If $N \geq \max \{Q-K, R-L\}$ and $\Re\left(\gamma_{q}\right), \Re\left(\delta_{r}\right)>0$, then

$$
\begin{aligned}
& \int_{U(N)} \frac{\prod_{k=1}^{K} \Lambda_{A}\left(\mathrm{e}^{-\alpha_{j}}\right) \prod_{\ell=K+1}^{K+L} \Lambda_{A^{*}}\left(\mathrm{e}^{\alpha_{\ell}}\right)}{\prod_{q=1}^{Q} \Lambda_{A}\left(\mathrm{e}^{-\gamma_{q}}\right) \prod_{r=1}^{R} \Lambda_{A^{*}}\left(\mathrm{e}^{-\delta_{r}}\right)} d A \\
&= \sum_{\sigma \in \Xi_{K, L}} \mathrm{e}^{N \sum_{k=1}^{K}\left(\alpha_{\sigma(k)}-\alpha_{k}\right)} \\
& \quad \times \frac{\prod_{k=1}^{K} \prod_{\ell=K+1}^{K+L} z\left(\alpha_{\sigma(k)}-\alpha_{\sigma(\ell)}\right) \prod_{q=1}^{Q} \prod_{r=1}^{R} z\left(\gamma_{q}+\delta_{r}\right)}{\prod_{r+1}^{R} \prod_{k=1}^{K} z\left(\alpha_{\sigma(k)}+\delta_{r}\right) \prod_{q=1}^{Q} \prod_{\ell=K+1}^{K+L} z\left(\gamma_{q}-\alpha_{\sigma(\ell)}\right)}
\end{aligned}
$$

If we let

$$
y_{U}(\alpha ; \beta ; \gamma ; \delta):=\frac{\prod_{k=1}^{K} \prod_{\ell=1}^{L} z\left(\alpha_{k}+\beta_{\ell}\right) \prod_{q=1}^{Q} \prod_{r=1}^{R} z\left(\gamma_{q}+\delta_{r}\right)}{\prod_{k=1}^{K} \prod_{r=1}^{R} z\left(\alpha_{k}+\delta_{r}\right) \prod_{\ell=1}^{L} \prod_{q=1}^{Q} z\left(\beta_{\ell}+\gamma_{q}\right)},
$$

then the above can be expressed as

$$
\begin{aligned}
& \int_{U(N)} \frac{\prod_{k=1}^{K} \Lambda_{A}\left(\mathrm{e}^{-\alpha_{k}}\right) \prod_{\ell=K+1}^{K+L} \Lambda_{A^{*}}\left(\mathrm{e}^{\alpha_{\ell}}\right)}{\prod_{q=1}^{Q} \Lambda_{A}\left(\mathrm{e}^{-\gamma_{q}}\right) \prod_{r=1}^{R} \Lambda_{A^{*}}\left(\mathrm{e}^{-\delta_{r}}\right)} d A \\
&= \sum_{\sigma \in \Xi_{K, L}} \mathrm{e}^{N \sum_{k=1}^{K}\left(\alpha_{\sigma(k)}-\alpha_{k}\right)} \\
& \quad \times y_{U}\left(\alpha_{\sigma(1)}, \ldots, \alpha_{\sigma(K)} ;-\alpha_{\sigma(K+1)} \cdots-\alpha_{\sigma(K+L)} ; \gamma ; \delta\right) .
\end{aligned}
$$

Theorem 4.2. If $2 N \geq Q-K-1$ and $\Re\left(\gamma_{q}\right)>0$, then

$$
\begin{aligned}
\int_{U \operatorname{Sp}(2 N)} & \frac{\prod_{k=1}^{K} \Lambda_{A}\left(\mathrm{e}^{-\alpha_{k}}\right)}{\prod_{q=1}^{Q} \Lambda_{A}\left(\mathrm{e}^{-\gamma_{q}}\right)} d A \\
= & \sum_{\epsilon \in\{-1,1\}^{K}} \mathrm{e}^{N \sum_{k=1}^{K}\left(\epsilon_{k} \alpha_{k}-\alpha_{k}\right)} \\
& \times \frac{\prod_{j \leq k \leq K} z\left(\epsilon_{j} \alpha_{j}+\epsilon_{k} \alpha_{k}\right) \prod_{q<r \leq Q} z\left(\gamma_{q}+\gamma_{r}\right)}{\prod_{k=1}^{K} \prod_{q=1}^{Q} z\left(\epsilon_{k} \alpha_{k}+\gamma_{q}\right)}
\end{aligned}
$$


If we let

$$
y_{S}(\alpha ; \gamma):=\frac{\prod_{j \leq k \leq K} z\left(\alpha_{j}+\alpha_{k}\right) \prod_{q<r \leq Q} z\left(\gamma_{q}+\gamma_{r}\right)}{\prod_{k=1}^{K} \prod_{q=1}^{Q} z\left(\alpha_{k}+\gamma_{q}\right)}
$$

and

$$
h_{S}(\alpha ; \gamma)=\mathrm{e}^{N \sum_{k=1}^{K} \epsilon_{k} \alpha_{k}} y_{S}(\alpha ; \gamma)
$$

then the above can be expressed as

$\int_{U \mathrm{Sp}(2 N)} \frac{\prod_{k=1}^{K} \Lambda_{A}\left(\mathrm{e}^{-\alpha_{k}}\right)}{\prod_{q=1}^{Q} \Lambda_{A}\left(\mathrm{e}^{-\gamma_{q}}\right)} d A=\mathrm{e}^{-N \sum_{k=1}^{K} \alpha_{k}} \sum_{\epsilon \in\{-1,1\}^{K}} h_{S}\left(\epsilon_{1} \alpha_{1}, \ldots, \epsilon_{K} \alpha_{K} ; \gamma\right)$

Theorem 4.3. If $2 N \geq Q-K+1$ and $\Re\left(\gamma_{q}\right)>0$, then

$$
\begin{aligned}
& \int_{\mathrm{SO}(2 N)} \frac{\prod_{k=1}^{K} \Lambda_{A}\left(\mathrm{e}^{-\alpha_{k}}\right)}{\prod_{q=1}^{Q} \Lambda_{A}\left(\mathrm{e}^{-\gamma_{q}}\right)} d A \\
& \quad=\sum_{\epsilon \in\{-1,1\}^{K}} \mathrm{e}^{N \sum_{k=1}^{K}\left(\epsilon_{k} \alpha_{k}-\alpha_{k}\right)} \frac{\prod_{j<k \leq K} z\left(\epsilon_{j} \alpha_{j}+\epsilon_{k} \alpha_{k}\right) \prod_{q \leq r \leq Q} z\left(\gamma_{q}+\gamma_{r}\right)}{\prod_{k=1}^{K} \prod_{r=1}^{R} z\left(\epsilon_{k} \alpha_{k}+\gamma_{r}\right)}
\end{aligned}
$$

If we let

$$
y_{O}(\alpha ; \gamma):=\frac{\prod_{j<k \leq K} z\left(\alpha_{j}+\alpha_{k}\right) \prod_{q \leq r \leq Q} z\left(\gamma_{q}+\gamma_{r}\right)}{\prod_{k=1}^{K} \prod_{q=1}^{Q} z\left(\alpha_{k}+\gamma_{q}\right)}
$$

and

$$
h_{O}(\alpha ; \gamma)=\mathrm{e}^{N \sum_{k=1}^{K} \epsilon_{k} \alpha_{k}} y_{O}(\alpha ; \gamma)
$$

then the above can be expressed as

$$
\int_{\mathrm{SO}(2 N)} \frac{\prod_{k=1}^{K} \Lambda_{A}\left(\mathrm{e}^{-\alpha_{k}}\right)}{\prod_{q=1}^{Q} \Lambda_{A}\left(\mathrm{e}^{-\gamma_{q}}\right)} d A=\mathrm{e}^{-N \sum_{k=1}^{K} \alpha_{k}} \sum_{\epsilon \in\{-1,1\}^{K}} h_{O}\left(\epsilon_{1} \alpha_{1}, \ldots, \epsilon_{K} \alpha_{K} ; \gamma\right) .
$$


Theorem 4.4. If $2 N \geq Q-K$ and $\Re\left(\gamma_{q}\right)>0$, then

$$
\begin{aligned}
\int_{\mathrm{SO}(2 N+1)} \frac{\prod_{k=1}^{K} \Lambda_{A}\left(\mathrm{e}^{-\alpha_{k}}\right)}{\prod_{q=1}^{Q} \Lambda_{A}\left(\mathrm{e}^{-\gamma_{q}}\right)} d A \\
=\sum_{\epsilon \in\{-1,1\}^{K}} \operatorname{sgn}(\epsilon) \mathrm{e}^{N \sum_{k=1}^{K}\left(\epsilon_{k} \alpha_{k}-\alpha_{k}\right)} \\
\quad \times \frac{\prod_{j<k \leq K} z\left(\epsilon_{j} \alpha_{j}+\epsilon_{k} \alpha_{k}\right) \prod_{q \leq r \leq Q} z\left(\gamma_{q}+\gamma_{r}\right)}{\prod_{k=1}^{K} \prod_{q=1}^{Q} z\left(\epsilon_{k} \alpha_{k}+\gamma_{q}\right)} \\
=\mathrm{e}^{-N \sum_{k=1}^{K} \alpha_{k}} \sum_{\epsilon \in\{-1,1\}^{K}} \operatorname{sgn}(\epsilon) h_{O}\left(\epsilon_{1} \alpha_{1}, \ldots, \epsilon_{K} \alpha_{K} ; \gamma\right) .
\end{aligned}
$$

In the next section, we give conjectures for the averages of ratios of $L$-functions, which will have a very similar form to the theorems given above.

\section{Conjectures about autocorrelations of ratios of $L$-functions}

We make conjectures about averages of $L$-functions which are analogous to the theorems of the previous section. Roughly speaking, the number $N$ of independent eigenvalues of the matrix is replaced by the analytic conductor of the family, the function $z(x)$ in the above theorems is replaced by $\zeta(1+x)$, and in addition, an arithmetic factor $A$ must be introduced. This arithmetic factor, which depends on the particular family under consideration, is expressible as an infinite product over primes and can be computed on a case by case basis.

In Section 5.1, we give our recipe for conjecturing averages of ratios, then we illustrate the computation for some standard examples of families of $L$-functions. For a more detailed discussion of related conjectures, see $[6]$.

\subsection{The recipe}

The following is an extension of the approximate functional equation recipe of [6]. Familiarity with that paper will be helpful here. 
Suppose $\mathcal{L}$ is an $L$-function and $\mathcal{F}=\{f\}$ is a family of characters with conductor $c(f)$, as described in Section 3 of [6]. Thus, $\mathcal{L}(s, f)$ has an approximate functional equation of the form

$$
\mathcal{L}(s, f)=\sum \frac{a_{n}(f)}{n^{s}}+\varepsilon_{f} \mathcal{X}_{f}(s) \sum \frac{\overline{a_{n}(f)}}{n^{1-s}}+\text { remainder }
$$

Here $\varepsilon_{f}$ is the sign in the functional equation and $\mathcal{X}_{f}$ is the ratio of $\Gamma$-functions that appears in the asymmetric form of the functional equation for $\mathcal{L}(s, f)$. Also, we can write

$$
\frac{1}{\mathcal{L}(s, f)}=\sum_{n=1}^{\infty} \frac{\mu_{\mathcal{L}, f}(n)}{n^{s}},
$$

the series converging absolutely for $\Re(s)>1$ and conditionally, assuming a suitable Riemann hypothesis, for $\Re(s)>\frac{1}{2}$.

We wish to conjecture a precise asymptotic formula for the average

$$
\begin{aligned}
& \mathcal{L}\left(1 / 2+\alpha_{1}, f\right) \cdots \mathcal{L}\left(1 / 2+\alpha_{K}, f\right) \\
& \sum_{f \in \mathcal{F}} \frac{\times \mathcal{L}\left(1 / 2+\alpha_{K+1}, \bar{f}\right) \cdots \mathcal{L}\left(1 / 2+\alpha_{K+L}, \bar{f}\right)}{\quad \begin{array}{l}
\mathcal{L}\left(1 / 2+\gamma_{1}, f\right) \cdots \mathcal{L}\left(1 / 2+\gamma_{Q}, f\right) \\
\times \mathcal{L}\left(1 / 2+\delta_{1}, \bar{f}\right) \cdots \mathcal{L}\left(1 / 2+\delta_{R}, \bar{f}\right)
\end{array}} g(c(f))
\end{aligned}
$$

where $g$ is a suitable test function. Note that the sum is an integral in the case of moments in $t$-aspect.

The recipe:

1. Start with

$$
\begin{aligned}
\mathcal{L}_{f}\left(s ; \boldsymbol{\alpha}_{K} ; \boldsymbol{\alpha}_{L} ; \gamma_{Q} ; \boldsymbol{\delta}_{R}\right) \\
\quad=\frac{\mathcal{L}\left(s+\alpha_{1}, f\right) \cdots \mathcal{L}\left(s+\alpha_{K}, f\right) \mathcal{L}\left(s+\alpha_{K+1}, \bar{f}\right) \cdots \mathcal{L}\left(s+\alpha_{K+L}, \bar{f}\right)}{\mathcal{L}\left(s+\gamma_{1}, f\right) \cdots \mathcal{L}\left(s+\gamma_{Q}, f\right) \mathcal{L}\left(s+\delta_{1}, \bar{f}\right) \cdots \mathcal{L}\left(s+\delta_{R}, \bar{f}\right)} .
\end{aligned}
$$

2. Replace each $L$-function in the numerator with the two terms from its approximate functional equation (5.1), ignoring the remainder term. Replace each $L$-function in the denominator by its series (5.2). Multiply out the resulting expression to obtain $2^{K+L}$ terms. Write those terms as

$$
\text { (product of } \varepsilon_{f} \text { factors)(product of } \mathcal{X}_{f} \text { factors) } \sum_{n_{1}, \ldots, n_{K+L+Q+R}} \text { (summand). }
$$


3. Replace each product of $\varepsilon_{f}$-factors by its expected value when averaged over the family.

4. Replace each summand by its expected value when averaged over the family.

5. Complete the resulting sums (i.e., extend the ranges of the summation indices out to infinity), and call the total $M_{f}\left(s, \boldsymbol{\alpha}_{K} ; \boldsymbol{\alpha}_{L} ; \boldsymbol{\gamma}_{Q} ; \boldsymbol{\delta}_{R}\right)$.

6. The conjecture is

$$
\begin{aligned}
& \sum_{f \in \mathcal{F}} \mathcal{L}_{f}\left(\frac{1}{2}, \boldsymbol{\alpha}_{K} ; \boldsymbol{\alpha}_{L} ; \boldsymbol{\gamma}_{Q} ; \boldsymbol{\delta}_{R}\right) g(c(f)) \\
& \quad=\sum_{f \in \mathcal{F}} M_{f}\left(\frac{1}{2}, \boldsymbol{\alpha}_{K} ; \boldsymbol{\alpha}_{L} ; \boldsymbol{\gamma}_{Q} ; \boldsymbol{\delta}_{R}\right)\left(1+O\left(\mathrm{e}^{(-(1 / 2)+\varepsilon) c(f)}\right)\right) g(c(f))
\end{aligned}
$$

for all $\varepsilon>0$, where $g$ is a suitable weight function.

In other words, $\mathcal{L}_{f}\left(\frac{1}{2}, \cdot\right)$ and $M_{f}\left(\frac{1}{2}, \cdot\right)$ have the same value distribution if averaged over a sufficiently large portion of the family. Note that the dependence of $M_{f}$ on $f$ only occurs in the product of $\mathcal{X}_{f}$ factors.

The above conjecture has a square-root error term. Presumably this is best possible. Since very little is known about mean values of ratios, we are not able to give any objective evidence for such a small error term. Also, we have not specified the allowable range for the shifts $\alpha, \gamma$ and $\delta$. Conrey and Snaith [10] suggest that in the case of the zeta-function one should allow shifts with imaginary part $\ll T^{1-\epsilon}$.

\subsection{Moments of ratios of $\zeta(s)$}

Let $s=1 / 2+i t$ and consider

$$
\frac{1}{T} \int_{0}^{T} \frac{\prod_{k=1}^{K} \zeta\left(s+\alpha_{k}\right) \prod_{\ell=K+1}^{K+L} \zeta\left(1-s-\alpha_{\ell}\right)}{\prod_{q=1}^{Q} \zeta\left(s+\gamma_{q}\right) \prod_{r=1}^{R} \zeta\left(1-s+\delta_{r}\right)} d t
$$

where $\Re \alpha_{k}, \gamma_{q}, \delta_{r}>0$. We assume the Riemann hypothesis, so there are no poles on the path of integration. We follow the recipe given above.

First replace each $\zeta$-function in the numerator by its approximate functional equation

$$
\zeta(s) \sim \sum_{n \leq \tau} \frac{1}{n^{s}}+\chi(s) \sum_{n \leq \tau} \frac{1}{n^{1-s}}
$$


(where $\tau=\sqrt{t /(2 \pi)}$ ). Here $\chi$ is the factor in the functional equation, given in (3.4). Second, multiply out to get $2^{K+L}$ terms. Only retain the terms in which the same number of $\chi(s)$ as $\chi(1-s)$ occurs, because the others are highly oscillatory and have expected value 0 when averaged over $t$. For the terms in the denominator, expand into series

$$
\frac{1}{\zeta(s)}=\prod_{p}\left(1-\frac{1}{p^{s}}\right)=\sum_{n=1}^{\infty} \frac{\mu(n)}{n^{s}} .
$$

Here $\mu(n)$ is the Möbius function, which is multiplicative and is equal to -1 when $n=p$ is prime and is 0 when $n=p^{e}$ where $e>1$.

For each of the retained terms, we keep those summands which are independent of the parameter $t$; equivalently, we keep the "diagonal". (The other summands are of the form $\Theta^{t}$ with $\Theta \neq 1$, which has 0 expected value.)

In these calculations, it is easiest to initially work with the expression obtained from the 'first' term of each approximate functional equation, and then modify that expression to obtain the complete main term. This will determine the arithmetic factor $A$ which appears in the $L$-function averages (but not in the analogous random matrix averages). For convenience, let $\beta_{\ell}=-\alpha_{K+\ell}$; moreover, assume that the real parts of all the variables $\alpha_{k}, \beta_{\ell}, \gamma_{q}, \delta_{r}$ are positive so that the series

$$
\begin{aligned}
G_{\zeta}(\alpha ; \beta ; \gamma ; \delta)= & \sum_{\prod m_{k} \prod h_{q}=\prod n_{\ell} \prod j_{r}} \\
& \times \frac{\prod \mu\left(h_{q}\right) \prod \mu\left(j_{r}\right)}{\prod m_{k}^{1 / 2+\alpha_{k}} \prod n_{\ell}^{1 / 2+\beta_{\ell}} \prod h_{q}^{1 / 2+\gamma_{q}} \prod j_{r}^{1 / 2+\delta_{r}}}
\end{aligned}
$$

is absolutely convergent. We express $G_{\zeta}$ as an Euler product

$$
\begin{aligned}
G_{\zeta}(\alpha ; \beta ; \gamma ; \delta)= & \prod_{p} \sum_{\sum a_{k}+\sum c_{q}=\sum b_{\ell}+\sum d_{r}} \\
& \times \frac{\prod \mu\left(p^{c_{q}}\right) \prod \mu\left(p^{d_{r}}\right)}{\prod p^{\sum_{k}\left(1 / 2+\alpha_{k}\right) a_{k}+\sum_{\ell}\left(1 / 2+\beta_{\ell}\right) b_{\ell}+\sum_{q}\left(1 / 2+\gamma_{q}\right) c_{q}+\sum_{r}\left(1 / 2+\delta_{r}\right) d_{r}}} .
\end{aligned}
$$

The terms here with $\sum_{k=1}^{K} a_{k}+\sum_{q=1}^{Q} c_{q}=1=\sum_{\ell=1}^{n} b_{\ell}+\sum_{r=1}^{R} d_{r}$ contribute the zeros and poles. These terms give

$$
Y_{U}(\alpha ; \beta ; \gamma ; \delta):=\frac{\prod_{k=1}^{K} \prod_{\ell=1}^{L} \zeta\left(1+\alpha_{k}+\beta_{\ell}\right) \prod_{q=1}^{Q} \prod_{r=1}^{R} \zeta\left(1+\gamma_{q}+\delta_{r}\right)}{\prod_{k=1}^{K} \prod_{r=1}^{R} \zeta\left(1+\alpha_{k}+\delta_{r}\right) \prod_{\ell=1}^{L} \prod_{q=1}^{Q} \zeta\left(1+\beta_{\ell}+\gamma_{q}\right)} .
$$


We factor $Y_{U}$ out of $G_{\zeta}$ and are left with

$$
G_{\zeta}(\alpha ; \beta ; \gamma ; \delta)=Y_{U}(\alpha ; \beta ; \gamma ; \delta) A_{\zeta}(\alpha ; \beta ; \gamma ; \delta)
$$

where $A_{\zeta}$ is an Euler product, absolutely convergent for all of the variables in small disks around 0 , which is given by

$$
\begin{aligned}
A_{\zeta}= & \prod_{p} \frac{\prod_{K=1}^{K} \prod_{\ell=1}^{L}\left(1-1 / p^{1+\alpha_{k}+\beta_{\ell}}\right) \prod_{q=1}^{Q} \prod_{r=1}^{R}\left(1-1 / p^{1+\gamma_{q}+\delta_{r}}\right)}{\prod_{k=1}^{K} \prod_{r=1}^{R}\left(1-1 / p^{1+\alpha_{k}+\delta_{r}}\right) \prod_{\ell=1}^{L} \prod_{q=1}^{Q}\left(1-1 / p^{1+\beta_{\ell}+\gamma_{q}}\right)} \\
& \times \sum_{\sum a_{k}+\sum c_{q}=\sum b_{\ell}+\sum d_{r}} \\
& \times \frac{\prod \mu\left(p_{q}^{c}\right) \prod \mu\left(p^{d_{r}}\right)}{p^{\sum\left(1 / 2+\alpha_{k}\right) a_{k}+\sum\left(1 / 2+\beta_{\ell}\right) b_{\ell}+\sum\left(1 / 2+\gamma_{q}\right) c_{q}+\sum\left(1 / 2+\delta_{r}\right) d_{r}}} .
\end{aligned}
$$

Thus, the recipe leads us to:

Conjecture 5.1. If $\Re\left(\gamma_{q}\right), \Re\left(\delta_{r}\right)>0$ and $\Re\left(\alpha_{j}\right)>-\frac{1}{2(K+L)}$, then

$$
\begin{aligned}
\int_{0}^{T} & \frac{\prod_{k=1}^{K} \zeta\left(s+\alpha_{k}\right) \prod_{\ell=K+1}^{K+L} \zeta\left(1-s-\alpha_{\ell}\right)}{\prod_{q=1}^{Q} \zeta\left(s+\gamma_{q}\right) \prod_{r=1}^{R} \zeta\left(1-s+\delta_{r}\right)} d t \\
= & \int_{0}^{T} \sum_{\sigma \in \Xi_{K, L}} \prod_{k=1}^{K} \frac{\chi\left(s+\alpha_{k}\right)}{\chi\left(s-\alpha_{\sigma(k)}\right)} \\
& \times Y_{U} A_{\zeta}\left(\alpha_{\sigma(1)}, \ldots, \alpha_{\sigma(K)} ;-\alpha_{\sigma(K+1)} \cdots-\alpha_{\sigma(K+L)} ; \gamma ; \delta\right) d t \\
& +O\left(T^{1 / 2+\epsilon}\right) .
\end{aligned}
$$

Note that $\prod_{k=1}^{K+L} \chi\left(s+\alpha_{k}\right)=\prod_{k=1}^{K+L} \chi\left(s+\alpha_{\sigma(k)}\right)$ so that

$$
\prod_{k=1}^{K} \frac{\chi\left(s+\alpha_{k}\right)}{\chi\left(s-\alpha_{\sigma(k)}\right)}=\prod_{k=1}^{K} \frac{\chi\left(s+\alpha_{k}\right)^{1 / 2}}{\chi\left(s-\alpha_{\sigma(k)}\right)^{1 / 2}} \prod_{\ell=1}^{L} \frac{\chi\left(s-\alpha_{\sigma(K+\ell)}\right)^{1 / 2}}{\chi\left(s+\alpha_{K+\ell}\right)^{1 / 2}} .
$$

Thus, the factor on the left can be replaced by the factor on the right; this leads to a slightly different formulation of the conjecture which is convenient in Section 6.4 where we replace the combinatorial sum by a multiple integral. In particular, letting

$$
\begin{aligned}
H_{\zeta, t}\left(w_{1}, \ldots, w_{K+L} ; \gamma ; \delta\right)= & \frac{\prod_{\ell=1}^{L} \chi\left(s-w_{K+\ell}\right)^{1 / 2}}{\prod_{k=1}^{K} \chi\left(s+w_{k}\right)^{1 / 2}} \\
& \times Y_{U} A_{\zeta}\left(w_{1}, \ldots, w_{K} ;-w_{K+1}, \ldots,-w_{K+L} ; \gamma ; \delta\right)
\end{aligned}
$$


the conjecture may be reformulated as

$$
\begin{aligned}
\int_{0}^{T} & \frac{\prod_{k=1}^{K} \zeta\left(s+\alpha_{k}\right) \prod_{\ell=K+1}^{K+L} \zeta\left(1-s-\alpha_{\ell}\right)}{\prod_{q=1}^{Q} \zeta\left(s+\gamma_{q}\right) \prod_{r=1}^{R} \zeta\left(1-s+\delta_{r}\right)} d t \\
= & \int_{0}^{T} \frac{\prod_{k=1}^{K} \chi\left(s+\alpha_{k}\right)^{1 / 2}}{\prod_{\ell=1}^{L} \chi\left(s-\alpha_{K+\ell}\right)^{1 / 2}} \\
& \times \sum_{\sigma \in \Xi_{K, L}} H_{\zeta, t}\left(\alpha_{\sigma(1)}, \ldots, \alpha_{\sigma(K)} ; \alpha_{\sigma(K+1)} \cdots \alpha_{\sigma(K+L)} ; \gamma ; \delta\right) d t \\
& +O\left(T^{1 / 2+\epsilon}\right) .
\end{aligned}
$$

Conrey and Snaith [10] have an alternative formulation for this conjecture with a subscript-free notation.

\subsection{Moments of $L\left(1 / 2, \chi_{d}\right)$}

The family $\mathcal{D}^{+}=\left\{L\left(s, \chi_{d}\right): d>0\right\}$ is a symplectic family. We can make a conjecture analogous to Theorem 4.2 for

$$
\sum_{0<d \leq X} \frac{\prod_{k=1}^{K} L\left(1 / 2+\alpha_{k}, \chi_{d}\right)}{\prod_{q=1}^{Q} L\left(1 / 2+\gamma_{q}, \chi_{d}\right)}
$$

As in the previous example, the main issue will be identifying the appropriate arithmetic factor $A_{\mathcal{D}}$.

Again we follow the recipe, which will parallel the example of the Riemann $\zeta$-function in the previous section. The $L$-functions in the numerator are replaced by their approximate functional equations while the ones in the denominator are expanded into series

$$
\frac{1}{L\left(s, \chi_{d}\right)}=\prod_{p}\left(1-\frac{\chi_{d}(p)}{p^{s}}\right)=\sum_{n=1}^{\infty} \frac{\mu(n) \chi_{d}(n)}{n^{s}}
$$

with $\mu(n)$ as before.

Expanding the product of approximate functional equations, we obtain $2^{K}$ terms. All those terms are retained because the sign of the functional equation is always +1 . So now we replace the summands by their average. 
We can determine $G_{\mathcal{D}}$ (analogous to $G_{\zeta}$ in the previous example) by consideration of

$$
\begin{aligned}
G_{\mathcal{D}}(\alpha ; \gamma):= & \lim _{X \rightarrow \infty} \frac{1}{X^{*}} \sum_{m_{k}, h_{q}} \frac{\prod_{q} \mu\left(h_{q}\right)}{\prod_{k} m_{k}^{1 / 2+\alpha_{k}} \prod_{q} h_{q}^{1 / 2+\gamma_{q}}} \\
& \times \sum_{0<d \leq X} \chi_{d}\left(\prod_{k} m_{k} \prod_{q} h_{q}\right) \\
= & \sum_{m_{k}, h_{q}} \frac{\prod_{q} \mu\left(h_{q}\right)}{\prod_{k} m_{k}^{1 / 2+\alpha_{k}} \prod_{q} h_{q}^{1 / 2+\gamma_{q}}} \delta\left(\prod_{k} m_{k} \prod_{q} h_{q}\right)
\end{aligned}
$$

where $\delta(n)=\prod_{p \mid n}(1+1 / p)^{-1}$ if $n$ is a square and is 0 otherwise. We can express $G_{\mathcal{D}}$ as a convergent Euler product provided that the real parts of the $\alpha_{k}$ and the $\gamma_{q}$ are positive. Thus,

$$
\begin{aligned}
G_{\mathcal{D}}(\alpha ; \gamma)= & \prod_{p}\left(1+\left(1+\frac{1}{p}\right)^{-1} \sum_{0<\sum_{k} a_{k}+\sum_{q} c_{q} \text { is even }}\right. \\
& \left.\times \frac{\prod_{q} \mu\left(p^{c_{q}}\right)}{p^{\sum_{k} a_{k}\left(1 / 2+\alpha_{k}\right)+\sum_{q} c_{q}\left(1 / 2+\gamma_{q}\right)}}\right) .
\end{aligned}
$$

The terms here with $\sum_{k=1}^{K} a_{k}+\sum_{q=1}^{Q} c_{q}=2$ contribute the zeros and poles. Specifically, poles arise from terms $a_{j}=a_{k}=1$ with $1 \leq j<k \leq K$ and from terms $a_{k}=2$ with $1 \leq k \leq K$. Poles also arise from terms with $c_{q}=$ $c_{r}=1$ with $1 \leq q<r \leq Q$. Note that poles do not arise from terms with $c_{q}=2$ since $\mu\left(p^{2}\right)=0$. Zeros arise from terms with $a_{k}=1=c_{q}$ with $1 \leq k \leq K$ and $1 \leq q \leq Q$. The contribution, expressed in terms of zeta-functions, of all of these zero and polar terms is

$$
Y_{S}(\alpha ; \gamma):=\frac{\prod_{j \leq k \leq K} \zeta\left(1+\alpha_{j}+\alpha_{k}\right) \prod_{q<r \leq Q} \zeta\left(1+\gamma_{q}+\gamma_{r}\right)}{\prod_{k=1}^{K} \prod_{q=1}^{Q} \zeta\left(1+\alpha_{k}+\gamma_{q}\right)}
$$


When we factor $Y_{S}$ out from $G_{\mathcal{D}}$ we are left with an Euler product $A_{\mathcal{D}}(\alpha, \gamma)$ which is absolutely convergent for all of the variables in small disks around 0. Specifically,

$$
\begin{aligned}
A_{\mathcal{D}}(\alpha, \gamma)= & \prod_{p} \frac{\prod_{j \leq k \leq K}\left(1-1 / p^{1+\alpha_{j}+\alpha_{k}}\right) \prod_{q<r \leq Q}\left(1-1 / p^{1+\gamma_{q}+\gamma_{r}}\right)}{\prod_{k=1}^{K} \prod_{q=1}^{Q}\left(1-1 / p^{1+\alpha_{k}+\gamma_{q}}\right)} \\
& \times\left(\begin{array}{l}
1+\left(1+\frac{1}{p}\right)^{-1} \sum_{0<\sum_{k} a_{k}+\sum_{q} c_{q}} \text { is even } \\
\left.\frac{\prod_{q} \mu\left(p^{c_{q}}\right)}{p^{\sum_{k} a_{k}\left(1 / 2+\alpha_{k}\right)+\sum_{q} c_{q}\left(1 / 2+\gamma_{q}\right)}}\right)
\end{array}\right.
\end{aligned}
$$

The functional equation may be written as

$$
L\left(s, \chi_{d}\right)=\left(\frac{|d|}{\pi}\right)^{1 / 2-s} g_{+}(s) L\left(1-s, \chi_{d}\right),
$$

where

$$
g_{+}(s)=\frac{\Gamma((1-s) / 2)}{\Gamma(s / 2)}
$$

Note that $g_{+}(1 / 2)=1$. The analytic conductor of $L\left(s, \chi_{d}\right)$ is $\frac{|d|}{\pi}$ so that the role of $2 N$ in Theorem 4.2 is played by $2 N=\log \frac{|d|}{\pi}$. (There are some subtleties concerning the "conductor" here. See the discussion of Conjecture 1.5.3 in [6].) We are led to

Conjecture 5.2. Suppose that the real parts of $\alpha_{k}$ and $\gamma_{q}$ are positive. Then

$$
\begin{aligned}
& \sum_{0<d \leq X} \frac{\prod_{k=1}^{K} L\left(1 / 2+\alpha_{k}, \chi_{d}\right)}{\prod_{q=1}^{Q} L\left(1 / 2+\gamma_{q}, \chi_{d}\right)} \\
& =\sum_{0<d \leq X} \sum_{\epsilon \in\{-1,1\}^{K}}\left(\frac{|d|}{\pi}\right)^{1 / 2 \sum_{k=1}^{K}\left(\epsilon_{k} \alpha_{k}-\alpha_{k}\right)} \\
& \quad \times \prod_{k=1}^{K} g_{+}\left(\frac{1}{2}+\frac{\alpha_{k}-\epsilon_{k} \alpha_{k}}{2}\right) Y_{S} A_{\mathcal{D}}\left(\epsilon_{1} \alpha_{1}, \ldots, \epsilon_{K} \alpha_{K} ; \gamma\right) \\
& \quad+O\left(X^{1 / 2+\epsilon}\right) .
\end{aligned}
$$


If we let

$$
\begin{aligned}
H_{\mathcal{D}^{+}, d, \alpha, \gamma}(w)= & \left(\frac{|d|}{\pi}\right)^{1 / 2 \sum_{k=1}^{K} w_{k}} \prod_{k=1}^{K} g_{+}\left(\frac{1}{2}+\frac{\alpha_{k}-w_{k}}{2}\right) \\
& \times Y_{S} A_{\mathcal{D}}\left(w_{1}, \ldots, w_{k} ; \gamma\right),
\end{aligned}
$$

then the conjecture may be formulated as

$$
\begin{aligned}
& \sum_{0<d \leq X} \frac{\prod_{k=1}^{K} L\left(1 / 2+\alpha_{k}, \chi_{d}\right)}{\prod_{q=1}^{Q} L\left(1 / 2+\gamma_{q}, \chi_{d}\right)} \\
& =\sum_{0<d \leq X}\left(\frac{|d|}{\pi}\right)^{-1 / 2 \sum_{k=1}^{K} \alpha_{k}} \sum_{\epsilon \in\{-1,1\}^{K}} H_{\mathcal{D}^{+}, d, \alpha, \gamma}\left(\epsilon_{1} \alpha_{1}, \ldots, \epsilon_{K} \alpha_{K}\right) \\
& \quad+O\left(X^{1 / 2+\epsilon}\right) .
\end{aligned}
$$

A conjecture for the moments of ratios of $L\left(s, \chi_{d}\right)$ with $d<0$ can be analogously formulated; the only change is that $g_{+}$is replaced by

$$
g_{-}(s)=\frac{\Gamma((2-s) / 2)}{\Gamma((s+1) / 2)} .
$$

For a proof of a special case of this conjecture, see [29].

\subsection{Moments of $L_{E}\left(1 / 2, \chi_{d}\right)$}

Given an elliptic curve $E$, the family $E^{+}(\mathcal{D})=\left\{L_{E}\left(s, \chi_{d}\right): w\left(E_{d}\right)=+1\right\}$ is an even orthogonal family and $E^{-}(\mathcal{D})=\left\{L_{E}\left(s, \chi_{d}\right): w\left(E_{d}\right)=-1\right\}$ is an odd orthogonal family. We can formulate conjectures analogous to Theorems 4.3 and 4.4 for

$$
\sum_{\substack{|d| \leq X \\ w\left(E_{d}\right)=+1}} \frac{\prod_{k=1}^{K} L_{E}\left(1 / 2+\alpha_{k}, \chi_{d}\right)}{\prod_{q=1}^{Q} L_{E}\left(1 / 2+\gamma_{q}, \chi_{d}\right)}
$$

and for the same sum over $d$ with $w\left(E_{d}\right)=-1$, once we have identified the appropriate arithmetic factor $A_{E(\mathcal{D})}$. Suppose that the $L$-function associated with $E$ has level $M$. Let

$$
\frac{1}{L_{E}(s)}=\prod_{p}\left(1-\frac{\lambda(p)}{p^{s}}+\frac{\chi_{0}(p)}{p^{2 s}}\right)=\sum_{n=1}^{\infty} \frac{\mu_{E}(n)}{n^{s}},
$$

where $\chi_{0}$ is the principal character $\bmod M$. So $\mu_{E}(n)$ is a multiplicative function which is equal to $-\lambda(p)$ for $n=p$, is equal to $\chi_{0}(p)$ if $n=p^{2}$ and is 0 if $n=p^{e}$ with $e>2$. Let $X_{+}^{*}=\left|\left\{d:|d| \leq X, w\left(E_{d}\right)=1\right\}\right|$. 
As in the previous two examples, the calculation is fairly straightforward up to the point of computing the arithmetic factor. So we consider

$$
\begin{aligned}
G_{E(\mathcal{D})}(\alpha ; \gamma):= & \lim _{X \rightarrow \infty} \frac{1}{X_{+}^{*}} \sum_{m_{k}, h_{q}} \frac{\prod_{k} \lambda\left(m_{k}\right) \prod_{q} \mu_{E}\left(h_{q}\right)}{\prod_{k} m_{k}^{1 / 2+\alpha_{k}} \prod_{q} h_{q}^{1 / 2+\gamma_{q}}} \\
& \times \sum_{\substack{|d| \leq X \\
w\left(E_{d}\right)=1}} \chi_{d}\left(\prod_{k} m_{k} \prod_{q} h_{q}\right) \\
= & \sum_{m_{k}, h_{q}} \frac{\prod_{k} \lambda\left(m_{k}\right) \prod_{q} \mu_{E}\left(h_{q}\right)}{\prod_{k} m_{k}^{1 / 2+\alpha_{k}} \prod_{q} h_{q}^{1 / 2+\gamma_{q}} \delta} \delta\left(\prod_{k} m_{k} \prod_{q} h_{q}\right)
\end{aligned}
$$

where, as before, $\delta(n)=\prod_{p \mid n}(1+1 / p)^{-1}$ if $n$ is a square and is 0 otherwise. We can express $G_{E(\mathcal{D})}$ as a convergent Euler product provided that the real parts of the $\alpha_{k}$ and the $\gamma_{q}$ are positive. Thus,

$$
\begin{aligned}
G_{E(\mathcal{D})}(\alpha ; \gamma)= & \prod_{p}\left(1+\left(1+\frac{1}{p}\right)^{-1} \sum_{0<\sum_{k} a_{k}+\sum_{q} c_{q} \text { is even }}\right. \\
& \left.\times \frac{\prod_{k} \lambda\left(p^{a_{k}}\right) \prod_{q} \mu_{E}\left(p^{c_{q}}\right)}{p^{\sum_{k} a_{k}\left(1 / 2+\alpha_{k}\right)+\sum_{q} c_{q}\left(1 / 2+\gamma_{q}\right)}}\right)
\end{aligned}
$$

The terms with the smallest positive exponents, that is $\sum_{k=1}^{K} a_{k}+\sum_{q=1}^{Q}$ $c_{q}=2$, contribute the zeros and poles. Specifically, poles arise from terms $a_{j}=a_{k}=1$ with $1 \leq j<k \leq K$. Note that the terms with $a_{k}=2$ do not contribute poles; this is because the function

$$
\sum_{n=1}^{\infty} \frac{\lambda\left(n^{2}\right)}{n^{s}}
$$

is analytic at $s=1$. Poles also arise from terms with $c_{q}=c_{r}=1$ with $1 \leq$ $q<r \leq Q$. Zeros arise from terms with $a_{k}=1=c_{q}$ with $1 \leq k \leq K$ and $1 \leq q \leq Q$. Zeros also arise from terms with $c_{q}=2$ since $\mu_{E}\left(p^{2}\right)=1$. The contribution, expressed in terms of zeta-functions, of all of these zero and 
polar terms is

$$
\begin{aligned}
& Y_{O}(\alpha ; \gamma) \\
& :=\frac{\prod_{j<k \leq K} \zeta\left(1+\alpha_{j}+\alpha_{k}\right) \prod_{q<r \leq Q} \zeta\left(1+\gamma_{q}+\gamma_{r}\right) \prod_{q=1}^{Q} \zeta\left(1+2 \gamma_{q}\right)}{\prod_{k=1}^{K} \prod_{q=1}^{Q} \zeta\left(1+\alpha_{k}+\gamma_{q}\right)}
\end{aligned}
$$

When we factor $Y_{O}$ out from $G_{E(\mathcal{D})}$, we are left with an Euler product $A_{E(\mathcal{D})}(\alpha, \gamma)$ which is absolutely convergent for all of the variables in small disks around 0. Specifically,

$$
\begin{aligned}
& A_{E(\mathcal{D})}(\alpha, \gamma) \\
& =\prod_{p} \frac{\prod_{1 \leq j<k \leq K}\left(1-1 / p^{1+\alpha_{j}+\alpha_{k}}\right) \prod_{1 \leq q<r \leq Q}\left(1-1 / p^{1+\gamma_{q}+\gamma_{r}}\right)}{\prod_{k=1}^{K} \prod_{q=1}^{Q}\left(1-1 / p^{1+\alpha_{k}+\gamma_{q}}\right)} \\
& \quad \times\left(1+\left(1+\frac{1}{p}\right)^{-1} \sum_{0<\sum_{k} a_{k}+\sum_{q} c_{q} \text { is even }} \frac{\prod_{k} \lambda\left(p^{a_{k}}\right) \prod_{q} \mu_{E}\left(p^{c_{q}}\right)}{p^{\sum_{k} a_{k}\left(1 / 2+\alpha_{k}\right)+\sum_{q} c_{q}\left(1 / 2+\gamma_{q}\right)}}\right) .
\end{aligned}
$$

Note: when the above expression is evaluated, primes dividing $M$ contribute differently than primes not dividing $M$. These cases are analysed when we refine our expression for $A$ in Section 6.3.

The functional equation may be written as

$$
L_{E}\left(s, \chi_{d}\right)=w\left(E_{d}\right)\left(\frac{\sqrt{M}|d|}{2 \pi}\right)^{1-2 s} g(s) L_{E}\left(1-s, \chi_{d}\right),
$$

where

$$
g(s)=\frac{\Gamma(1-s)}{\Gamma(s)}
$$

Note that $g(1 / 2)=1$. The analytic conductor of $L_{E}\left(s, \chi_{d}\right)$ is $\frac{M|d|^{2}}{4 \pi^{2}}$ so that the role of $2 N$ in Theorem 4.2 is played by $2 N=\log \frac{M|d|^{2}}{4 \pi^{2}}$. (Again there are some subtleties concerning the "conductor". See the discussion of Conjecture 1.5.5 in [6].) We are led to 
Conjecture 5.3. Suppose that the real parts of $\alpha_{k}$ and $\gamma_{q}$ are positive. Then

$$
\begin{aligned}
& \sum_{\substack{|d| \leq X \\
w\left(E_{d}\right)=1}} \frac{\prod_{k=1}^{K} L_{E}\left(1 / 2+\alpha_{k}, \chi_{d}\right)}{\prod_{q=1}^{Q} L_{E}\left(1 / 2+\gamma_{q}, \chi_{d}\right)} \\
& =\sum_{\epsilon \in\{-1,1\}^{K}} Y_{O} A_{E(\mathcal{D})}\left(\epsilon_{1} \alpha_{1}, \ldots, \epsilon_{K} \alpha_{K} ; \gamma\right) \prod_{k=1}^{K} g\left(\frac{1}{2}+\frac{\alpha_{k}-\epsilon_{k} \alpha_{k}}{2}\right) \\
& \quad \times \sum_{\substack{|d| \leq X \\
w\left(E_{d}\right)=1}}\left(\frac{M|d|^{2}}{4 \pi^{2}}\right)^{1 / 2 \sum_{k=1}^{K}\left(\epsilon_{k} \alpha_{k}-\alpha_{k}\right)}+O\left(X^{1 / 2+\epsilon}\right) .
\end{aligned}
$$

A conjecture for the moments of ratios of $L_{E}\left(s, \chi_{d}\right)$ over those $d$ for which $w\left(E_{d}\right)=-1$ can be analogously formulated.

Conjecture 5.4. Suppose that the real parts of $\alpha_{k}$ and $\gamma_{q}$ are positive. Then

$$
\begin{aligned}
& \sum_{\substack{|d| \leq X \\
w\left(E_{d}\right)=-1}} \frac{\prod_{k=1}^{K} L_{E}\left(1 / 2+\alpha_{k}, \chi_{d}\right)}{\prod_{q=1}^{Q} L_{E}\left(1 / 2+\gamma_{q}, \chi_{d}\right)} \\
& =\sum_{\epsilon \in\{-1,1\}^{K}} \operatorname{sgn}(\epsilon) Y_{O} A_{E(\mathcal{D})}\left(\epsilon_{1} \alpha_{1}, \ldots, \epsilon_{K} \alpha_{K} ; \gamma\right) \prod_{k=1}^{K} g\left(\frac{1}{2}+\frac{\epsilon_{k} \alpha_{k}-\alpha_{k}}{2}\right) \\
& \quad \times \sum_{\substack{|d| \leq X \\
w\left(E_{d}\right)=-1}}\left(\frac{M|d|^{2}}{4 \pi^{2}}\right)^{1 / 2 \sum_{k=1}^{K}\left(\epsilon_{k} \alpha_{k}-\alpha_{k}\right)}+O\left(X^{1 / 2+\epsilon}\right) .
\end{aligned}
$$

\section{Refinements of the conjectures}

In this section, we refine our conjectures in two ways. We find closed form expressions for the Euler products $A$ and we express the combinatorial sums in our conjectures as residues of multiple integrals. This is similar to the treatment in [6].

\subsection{Closed form expressions for $A_{\zeta}$}

Let $e(\theta)=\mathrm{e}^{2 \pi i \theta}$ and let $\delta_{0}(n)$ be the function which is 1 when $n=0$ and is 0 otherwise. Then $\delta_{0}(n)=\int_{0}^{1} e(n \theta) d \theta$. In the formula for $A_{\zeta}$ we then replace 
the summation condition $\sum a_{k}+\sum c_{q}=\sum b_{\ell}+\sum d_{r}$ by

$$
\begin{aligned}
\delta_{0} & \left(\sum a_{k}+\sum c_{q}-\sum b_{\ell}-\sum d_{r}\right) \\
& =\int_{0}^{1} e\left(\left(\sum a_{k}+\sum c_{q}-\sum b_{\ell}-\sum d_{r}\right) \theta\right) d \theta .
\end{aligned}
$$

After summing the geometric series that arise, we deduce

Lemma 6.1. Let $e(\theta)=\mathrm{e}^{2 \pi i \theta}$. Then

$$
\sum_{\sum a_{k}+\sum c_{q}=\sum b_{\ell}+\sum d_{r}} \frac{\prod \mu\left(p_{q}^{c}\right) \prod \mu\left(p^{d_{r}}\right)}{p^{\sum\left(1 / 2+\alpha_{k}\right) a_{k}+\sum\left(1 / 2+\beta_{\ell}\right) b_{\ell}+\sum\left(1 / 2+\gamma_{q}\right) c_{q}+\sum\left(1 / 2+\delta_{r}\right) d_{r}}}
$$

$$
=\int_{0}^{1} \frac{\prod_{q=1}^{Q}\left(1-e(\theta) / p^{1 / 2+\gamma_{q}}\right) \prod_{r=1}^{R}\left(1-e(-\theta) / p^{1 / 2+\delta_{r}}\right)}{\prod_{k=1}^{K}\left(1-e(\theta) / p^{1 / 2+\alpha_{k}}\right) \prod_{\ell=1}^{L}\left(1-e(-\theta) / p^{1 / 2+\beta_{\ell}}\right)} d \theta .
$$

\section{Corollary 6.2.}

$$
\begin{aligned}
A_{\zeta}(\alpha ; \beta ; \gamma ; \delta) & \\
= & \prod_{p} \frac{\prod_{K=1}^{K} \prod_{\ell=1}^{L}\left(1-1 / p^{1+\alpha_{k}+\beta_{\ell}}\right) \prod_{q=1}^{Q} \prod_{r=1}^{R}\left(1-1 / p^{1+\gamma_{q}+\delta_{r}}\right)}{\prod_{k=1}^{K} \prod_{r=1}^{R}\left(1-1 / p^{1+\alpha_{k}+\delta_{r}}\right) \prod_{\ell=1}^{L} \prod_{q=1}^{Q}\left(1-1 / p^{1+\beta_{\ell}+\gamma_{q}}\right)} \\
& \times \int_{0}^{1} \frac{\prod_{q=1}^{Q}\left(1-e(\theta) / p^{1 / 2+\gamma_{q}}\right) \prod_{r=1}^{R}\left(1-e(-\theta) / p^{1 / 2+\delta_{r}}\right)}{\prod_{k=1}^{K}\left(1-e(\theta) / p^{1 / 2+\alpha_{k}}\right) \prod_{\ell=1}^{L}\left(1-e(-\theta) / p^{1 / 2+\beta_{\ell}}\right)} d \theta .
\end{aligned}
$$

\subsection{Closed form expressions for $\boldsymbol{A}_{\mathcal{D}}$}

Suppose that $f(x)=1+\sum_{n=1}^{\infty} u_{n} x^{n}$. Then

$$
\sum_{0<n \text { is even }} u_{n} x^{n}=\frac{1}{2}(f(x)+f(-x)-2)
$$


and

$$
1+\left(1+\frac{1}{p}\right)^{-1} \sum_{0<n \text { is even }} u_{n} x^{n}=\frac{1}{1+1 / p}\left(\frac{f(x)+f(-x)}{2}+\frac{1}{p}\right) .
$$

We apply this with

$$
f\left(\frac{1}{p}\right)=\sum_{a_{k}, c_{q}} \frac{\prod_{q} \mu\left(p^{c_{q}}\right)}{p^{\sum_{k} a_{k}\left(1 / 2+\alpha_{k}\right)+\sum_{q} c_{q}\left(1 / 2+\gamma_{q}\right)}}=\frac{\prod_{q=1}^{Q}\left(1-1 / p^{1 / 2+\gamma_{q}}\right)}{\prod_{k=1}^{K}\left(1-1 / p^{1 / 2+\alpha_{k}}\right)}
$$

to deduce

\section{Lemma 6.3.}

$$
\begin{aligned}
1+\left(1+\frac{1}{p}\right)^{-1} & \sum_{0<\sum_{k} a_{k}+\sum_{q} c_{q} \text { is even }} \frac{\prod_{q} \mu\left(p^{c_{q}}\right)}{p_{k}^{\sum_{k}\left(1 / 2+\alpha_{k}\right)+\sum_{q} c_{q}\left(1 / 2+\gamma_{q}\right)}} \\
& =\frac{1}{1+1 / p}\left(\frac{1}{2} \frac{\prod_{q=1}^{Q}\left(1-1 / p^{1 / 2+\gamma_{q}}\right)}{\prod_{k=1}^{K}\left(1-1 / p^{1 / 2+\alpha_{k}}\right)}+\frac{1}{2} \frac{\prod_{q=1}^{Q}\left(1+1 / p^{1 / 2+\gamma_{q}}\right)}{\prod_{k=1}^{K}\left(1+1 / p^{1 / 2+\alpha_{k}}\right)}+\frac{1}{p}\right) .
\end{aligned}
$$

\section{Corollary 6.4.}

$$
\begin{aligned}
A_{\mathcal{D}}(\alpha, \gamma)= & \prod_{p} \frac{\prod_{j \leq k \leq K}\left(1-1 / p^{1+\alpha_{j}+\alpha_{k}}\right) \prod_{q<r \leq Q}\left(1-1 / p^{1+\gamma_{q}+\gamma_{r}}\right)}{\prod_{k=1}^{K} \prod_{q=1}^{Q}\left(1-1 / p^{1+\alpha_{k}+\gamma_{q}}\right)} \\
& \times \frac{1}{1+1 / p}\left(\frac{1}{2} \frac{\prod_{q=1}^{Q}\left(1-1 / p^{1 / 2+\gamma_{q}}\right)}{\prod_{k=1}^{K}\left(1-1 / p^{1 / 2+\alpha_{k}}\right)}\right. \\
& \left.+\frac{1}{2} \frac{\prod_{q=1}^{Q}\left(1+1 / p^{1 / 2+\gamma_{q}}\right)}{\prod_{k=1}^{K}\left(1+1 / p^{1 / 2+\alpha_{k}}\right)}+\frac{1}{p}\right)
\end{aligned}
$$

\subsection{Closed form expressions for $A_{E(\mathcal{D})}$}

For simplicity, let $E=E_{11}$. We apply the method of the last section, this time with

$$
f\left(\frac{1}{p}\right)=\sum_{a_{k}, c_{q}} \frac{\prod_{k} \lambda\left(p^{a_{k}}\right) \prod_{q} \mu_{E}\left(p^{c_{q}}\right)}{p^{\sum_{k} a_{k}\left(1 / 2+\alpha_{k}\right)+\sum_{q} c_{q}\left(1 / 2+\gamma_{q}\right)}} .
$$


If $p$ is not 11 , then

$$
f\left(\frac{1}{p}\right)=\frac{\prod_{q=1}^{Q}\left(1-\lambda(p) / p^{1 / 2+\gamma_{q}}+1 / p^{1+2 \gamma_{q}}\right)}{\prod_{k=1}^{K}\left(1-\lambda(p) / p^{1 / 2+\alpha_{k}}+\lambda(p) / p^{1+2 \alpha_{k}}\right)},
$$

whereas if $p=11$, then

$$
f\left(\frac{1}{p}\right)=\frac{\prod_{q=1}^{Q}\left(1-1 / \sqrt{11} / 11^{1 / 2+\gamma_{q}}\right)}{\prod_{k=1}^{K}\left(1-1 / \sqrt{11} / 11^{1 / 2+\alpha_{k}}\right)} .
$$

Lemma 6.5. If $p \neq 11$, then

$$
\begin{aligned}
& 1+\left(1+\frac{1}{p}\right)^{-1} \sum_{0<\sum_{k} a_{k}+\sum_{q} c_{q} \text { is even }} \frac{\prod_{k} \lambda\left(p^{a_{k}}\right) \prod_{q} \mu_{E}\left(p^{c_{q}}\right)}{p^{\sum_{k} a_{k}\left(1 / 2+\alpha_{k}\right)+\sum_{q} c_{q}\left(1 / 2+\gamma_{q}\right)}} \\
&= \frac{1}{1+1 / p}\left(\frac{1}{2} \frac{\prod_{q=1}^{Q}\left(1-\lambda(p) / p^{1 / 2+\gamma_{q}}+1 / p^{1+2 \gamma_{q}}\right)}{\prod_{k=1}^{K}\left(1-1 / p^{1 / 2+\alpha_{k}}+\lambda(p) / p^{1+2 \alpha_{k}}\right)}\right. \\
&\left.+\frac{1}{2} \frac{\prod_{q=1}^{Q}\left(1+\lambda(p) / p^{1 / 2+\gamma_{q}}+1 / p^{1+2 \gamma_{q}}\right)}{\prod_{k=1}^{K}\left(1+\lambda(p) / p^{1 / 2+\alpha_{k}}+\lambda(p) / p^{1+2 \alpha_{k}}\right)}+\frac{1}{p}\right) .
\end{aligned}
$$

while if $p=11$, then

$$
\begin{aligned}
1+ & \left(1+\frac{1}{p}\right)^{-1} \sum_{0<\sum_{k}} \sum_{a_{k}+\sum_{q} c_{q} \text { is even }} \frac{\prod_{k} \lambda\left(p^{a_{k}}\right) \prod_{q} \mu_{E}\left(p^{c_{q}}\right)}{p^{\sum_{k} a_{k}\left(1 / 2+\alpha_{k}\right)+\sum_{q} c_{q}\left(1 / 2+\gamma_{q}\right)}} \\
= & \frac{1}{1+1 / 11}\left(\frac{1}{2} \frac{\prod_{q=1}^{Q}\left(1-1 / \sqrt{11} / 11^{1 / 2+\gamma_{q}}\right)}{\prod_{k=1}^{K}\left(1-1 / \sqrt{11} / 11^{1 / 2+\alpha_{k}}\right)}\right. \\
& \left.+\frac{1}{2} \frac{\prod_{q=1}^{Q}\left(1+1 / \sqrt{11} / 11^{1 / 2+\gamma_{q}}\right)}{\prod_{k=1}^{K}\left(1+1 / \sqrt{11} / 11^{1 / 2+\alpha_{k}}\right)}+\frac{1}{11}\right) .
\end{aligned}
$$




\section{Corollary 6.6.}

$$
\begin{aligned}
A_{E_{11}(\mathcal{D})}(\alpha, \gamma)= & \frac{\prod_{1 \leq j<k \leq K}\left(1-1 / 11^{1+\alpha_{j}+\alpha_{k}}\right) \prod_{1 \leq q<r \leq Q}\left(1-1 / 11^{1+\gamma_{q}+\gamma_{r}}\right)}{\prod_{k=1}^{K} \prod_{q=1}^{Q}\left(1-1 / 11^{1+\alpha_{k}+\gamma_{q}}\right) \prod_{q=1}^{Q}\left(1-1 / 11^{1+2 \gamma_{q}}\right)} \\
& \times \frac{1}{1+1 / 11}\left(\frac{1}{2} \frac{\prod_{q=1}^{Q}\left(1-1 / \sqrt{11} / 11^{1 / 2+\gamma_{q}}\right)}{\prod_{k=1}^{K}\left(1-1 / \sqrt{11} / 11^{1 / 2+\alpha_{k}}\right)}\right. \\
& \left.+\frac{1}{2} \frac{\prod_{q=1}^{Q}\left(1+1 / \sqrt{11} / 11^{1 / 2+\gamma_{q}}\right)}{\prod_{k=1}^{K}\left(1+1 / \sqrt{11} / 11^{1 / 2+\alpha_{k}}\right)}+\frac{1}{11}\right) \\
& \times \prod_{p \neq 11} \frac{\prod_{1 \leq j<k \leq K}^{K}\left(1-1 / p^{1+\alpha_{j}+\alpha_{k}}\right) \prod_{1 \leq q<r \leq Q}\left(1-1 / p^{1+\gamma_{q}+\gamma_{r}}\right)}{\prod_{q=1}^{Q}\left(1-1 / p^{1+\alpha_{k}+\gamma_{q}}\right) \prod_{q=1}^{Q}\left(1-1 / p^{1+2 \gamma_{q}}\right)} \\
& \times \frac{1}{1+1 / p}\left(\frac{1}{2} \frac{\prod_{q=1}^{Q}\left(1-\lambda(p) / p^{1 / 2+\gamma_{q}}+1 / p^{1+2 \gamma_{q}}\right)}{\prod_{k=1}^{K}\left(1-1 / p^{1 / 2+\alpha_{k}}+\lambda(p) / p^{1+2 \alpha_{k}}\right)}\right. \\
& \left.+\frac{1}{2} \frac{\prod_{q=1}^{Q}\left(1+\lambda(p) / p^{1 / 2+\gamma_{q}}+1 / p^{1+2 \gamma_{q}}\right)}{\prod_{k=1}^{K}\left(1+\lambda(p) / p^{1 / 2+\alpha_{k}}+\lambda(p) / p^{1+2 \alpha_{k}}\right)}+\frac{1}{p}\right) . \\
(6.14) &
\end{aligned}
$$

\subsection{Combinatorial sums as integrals}

We express the sums appearing in our conjectures in terms of multiple integrals. The expressions will involve the Vandermonde determinant, given by

$$
\Delta\left(w_{1}, \ldots, w_{R}\right)=\operatorname{det}_{R \times R}\left(w_{i}^{j-1}\right) .
$$

We often omit the subscripts and write $\Delta(w)$ in place of $\Delta\left(w_{1}, \ldots, w_{R}\right)$. The key fact about the Vandermonde is that

$$
\Delta\left(w_{1}, \ldots, w_{R}\right)=\prod_{1 \leq j<k \leq R}\left(w_{j}-w_{i}\right) .
$$

Lemma 6.7. Suppose that $F(z ; w)=F\left(z_{1}, \ldots, z_{K} ; w_{1}, \ldots, w_{L}\right)$ is a function of $K+L$ variables, which is symmetric with respect to the first $K$ variables and symmetric with respect to the second set of $L$ variables. Suppose 
also that $F$ is regular near $(0, \ldots, 0)$. Suppose further that $f(s)$ has a simple pole of residue 1 at $s=0$ but is otherwise analytic in $|s| \leq 1$. Let

$$
H\left(z_{1}, \ldots, z_{K} ; w_{1}, \ldots, w_{L}\right)=F\left(z_{1}, \ldots ; \ldots, w_{L}\right) \prod_{k=1}^{K} \prod_{\ell=1}^{L} f\left(z_{k}-w_{\ell}\right) .
$$

If $\left|\alpha_{k}\right|<1$, then

$$
\begin{aligned}
& \sum_{\sigma \in \Xi_{K, L}} H\left(\alpha_{\sigma(1)}, \ldots, \alpha_{\sigma(K)} ; \alpha_{\sigma(K+1)} \ldots \alpha_{\sigma(K+L)}\right) \\
& =\frac{(-1)^{(K+L)(K+L-1) / 2}}{K ! L !(2 \pi i)^{K+L}} \\
& \quad \times \int_{\left|z_{i}\right|=1} \frac{H\left(z_{1}, \ldots, z_{K} ; z_{K+1}, \ldots, z_{K+L}\right) \Delta\left(z_{1}, \ldots, z_{K+L}\right)^{2}}{\prod_{j=1}^{K+L} \prod_{k=1}^{K+L}\left(z_{k}-\alpha_{j}\right)} d z_{1} \cdots d z_{K+L} .
\end{aligned}
$$

In view of the last formula of Section 6.1, and using

$$
\begin{aligned}
& H_{\zeta, t}\left(w_{1}, \ldots, w_{K+L} ; \gamma ; \delta\right) \\
& \quad=\frac{\prod_{\ell=1}^{L} \chi\left(s-w_{K+\ell}\right)^{1 / 2}}{\prod_{k=1}^{K} \chi\left(s+w_{k}\right)^{1 / 2}} Y_{U} A_{\zeta}\left(w_{1}, \ldots, w_{K} ;-w_{K+1}, \ldots,-w_{K+L} ; \gamma ; \delta\right)
\end{aligned}
$$

with

$$
\begin{aligned}
A_{\zeta}(\alpha ; \beta ; \gamma ; \delta) & \\
= & \prod_{p} \frac{\prod_{K=1}^{K} \prod_{\ell=1}^{L}\left(1-1 / p^{1+\alpha_{k}+\beta_{\ell}}\right) \prod_{q=1}^{Q} \prod_{r=1}^{R}\left(1-1 / p^{1+\gamma_{q}+\delta_{r}}\right)}{\prod_{k=1}^{K} \prod_{r=1}^{R}\left(1-1 / p^{1+\alpha_{k}+\delta_{r}}\right) \prod_{\ell=1}^{L} \prod_{q=1}^{Q}\left(1-1 / p^{1+\beta_{\ell}+\gamma_{q}}\right)} \\
& \times \int_{0}^{1} \frac{\prod_{q=1}^{Q}\left(1-e(\theta) / p^{1 / 2+\gamma_{q}}\right) \prod_{r=1}^{R}\left(1-e(-\theta) / p^{1 / 2+\delta_{r}}\right)}{\prod_{k=1}^{K}\left(1-e(\theta) / p^{1 / 2+\alpha_{k}}\right) \prod_{\ell=1}^{L}\left(1-e(-\theta) / p^{1 / 2+\beta_{\ell}}\right)} d \theta
\end{aligned}
$$

and

$$
Y_{U}(\alpha ; \beta ; \gamma ; \delta):=\frac{\prod_{k=1}^{K} \prod_{\ell=1}^{L} \zeta\left(1+\alpha_{k}+\beta_{\ell}\right) \prod_{q=1}^{Q} \prod_{r=1}^{R} \zeta\left(1+\gamma_{q}+\delta_{r}\right)}{\prod_{k=1}^{K} \prod_{r=1}^{R} \zeta\left(1+\alpha_{k}+\delta_{r}\right) \prod_{\ell=1}^{L} \prod_{q=1}^{Q} \zeta\left(1+\beta_{\ell}+\gamma_{q}\right)},
$$


we can reformulate Conjecture 5.1 as

$$
\begin{aligned}
\int_{0}^{T} & \frac{\prod_{k=1}^{K} \zeta\left(s+\alpha_{k}\right) \prod_{\ell=K+1}^{K+L} \zeta\left(1-s-\alpha_{\ell}\right)}{\prod_{q=1}^{Q} \zeta\left(s+\gamma_{q}\right) \prod_{r=1}^{R} \zeta\left(1-s+\delta_{r}\right)} d t \\
= & \int_{0}^{T} \frac{\prod_{k=1}^{K} \chi\left(s+\alpha_{k}\right)^{1 / 2}}{\prod_{\ell=1}^{L} \chi\left(s-\alpha_{K+\ell}\right)^{1 / 2}} \frac{(-1)^{(K+L)(K+L-1) / 2}}{K ! L !(2 \pi i)^{K+L}} \\
& \times \int_{\left|z_{i}\right|=1} \frac{H_{\zeta, t}\left(z_{1}, \ldots, z_{K} ; z_{K+1}, \ldots, z_{K+L}\right) \Delta\left(z_{1}, \ldots, z_{K+L}\right)^{2}}{\prod_{j=1}^{K+L} \prod_{k=1}^{K+L}\left(z_{k}-\alpha_{j}\right)} \\
& \times d z_{1} \cdots d z_{K+L} d t+O\left(T^{1 / 2+\epsilon}\right) .
\end{aligned}
$$

This should be compared with the reformulation of Theorem 4.1:

$$
\begin{aligned}
& \int_{U(N)} \frac{\prod_{k=1}^{K} \Lambda_{A}\left(\mathrm{e}^{-\alpha_{j}}\right) \prod_{\ell=K+1}^{K+L} \Lambda_{A^{*}}\left(\mathrm{e}^{\alpha_{\ell}}\right)}{\prod_{q=1}^{Q} \Lambda_{A}\left(\mathrm{e}^{-\gamma_{q}}\right) \prod_{r=1}^{R} \Lambda_{A^{*}}\left(\mathrm{e}^{-\delta_{r}}\right)} d A_{N} \\
&= \mathrm{e}^{N / 2\left(-\sum_{k=1}^{K} \alpha_{k}+\sum_{\ell=1}^{L} \alpha_{K+\ell}\right)} \frac{(-1)^{(K+L)(K+L-1) / 2}}{K ! L !(2 \pi i)^{K+L}} \\
& \quad \times \int_{\left|z_{i}\right|=1} \frac{H_{U}\left(z_{1}, \ldots, z_{K} ; z_{K+1}, \ldots, z_{K+L} ; \gamma ; \delta\right) \Delta\left(z_{1}, \ldots, z_{K+L}\right)^{2}}{\prod_{j=1}^{K+L} \prod_{k=1}^{K+L}\left(z_{k}-\alpha_{j}\right)} \prod_{k} d z_{k}
\end{aligned}
$$

where

$$
H_{U}\left(w_{1}, \ldots, w_{K+L} ; \gamma ; \delta\right)
$$

$$
=\mathrm{e}^{N / 2 \sum_{k=1}^{K} w_{k}-N / 2 \sum_{\ell=1}^{L} w_{K+\ell}} y_{U}\left(w_{1}, \ldots, w_{K} ;-w_{K+1}, \ldots,-w_{K+L} ; \gamma ; \delta\right) .
$$

Note that for a small shift $\alpha$,

$$
\chi(s+\alpha)=\left(\frac{|t|}{2 \pi}\right)^{1 / 2-s-\alpha}(1+O(1 /(1+|t|))
$$

so that, for example,

$$
\begin{aligned}
& \prod_{k=1}^{K} \frac{\chi\left(s+\alpha_{k}\right)^{1 / 2}}{\chi(s)^{1 / 2}} \prod_{\ell=1}^{L} \frac{\chi\left(s-\alpha_{K+\ell}\right)^{1 / 2}}{\chi(s)^{1 / 2}} \\
& \quad=\mathrm{e}^{\ell / 2\left(-\sum_{k=1}^{K} \alpha_{k}+\sum_{\ell=1}^{L} \alpha_{K+\ell}\right)}(1+O(1 /(1+|t|))
\end{aligned}
$$


where $\ell=\log \frac{t}{2 \pi}$, which compares with the random matrix formula with $N$ replaced by $\ell$. For large shifts, this approximation deteriorates and necessitates that we retain the accurate expression involving the product of $\chi$.

Note also that this integral formula gives an analytic continuation in the variables $\alpha$ and $\beta$ (so that we no longer need to restrict them to have positive real parts), whereas the variables $\gamma$ and $\delta$ are still required to have positive real parts.

Lemma 6.8. Suppose that $F(z)=F\left(z_{1}, \ldots, z_{K}\right)$ is a function of $K$ variables, which is symmetric and regular near $(0, \ldots, 0)$. Suppose further that $f(s)$ has a simple pole of residue 1 at $s=0$ but is otherwise analytic in $|s| \leq 1$. Let either

$$
H\left(z_{1}, \ldots, z_{K}\right)=F\left(z_{1}, \ldots, z_{K}\right) \prod_{1 \leq j \leq k \leq K} f\left(z_{j}+z_{k}\right)
$$

or

$$
H\left(z_{1}, \ldots, z_{K}\right)=F\left(z_{1}, \ldots, z_{K}\right) \prod_{1 \leq j<k \leq K} f\left(z_{j}+z_{k}\right)
$$

If $\left|\alpha_{k}\right|<1$, then

$$
\begin{aligned}
& \sum_{\epsilon \in\{-1,+1\}^{K}} H\left(\epsilon_{1} \alpha_{1}, \ldots, \epsilon_{K} \alpha_{K}\right) \\
& =\frac{(-1)^{K(K-1) / 2} 2^{K}}{K !(2 \pi i)^{K}} \int_{\left|z_{i}\right|=1} \frac{H\left(z_{1}, \ldots, z_{K}\right) \Delta\left(z_{1}^{2}, \ldots, z_{K}^{2}\right)^{2} \prod_{k=1}^{K} z_{k}}{\prod_{j=1}^{K} \prod_{k=1}^{K}\left(z_{k}-\alpha_{j}\right)\left(z_{k}+\alpha_{j}\right)} \\
& \quad \times d z_{1} \cdots d z_{K}
\end{aligned}
$$

and

$$
\begin{aligned}
& \sum_{\epsilon \in\{-1,+1\}^{K}} \operatorname{sgn}(\epsilon) H\left(\epsilon_{1} \alpha_{1}, \ldots, \epsilon_{K} \alpha_{K}\right) \\
& =\frac{(-1)^{K(K-1) / 2} 2^{K}}{K !(2 \pi i)^{K}} \int_{\left|z_{i}\right|=1} \frac{H\left(z_{1}, \ldots, z_{K}\right) \Delta\left(z_{1}^{2}, \ldots, z_{K}^{2}\right)^{2} \prod_{k=1}^{K} \alpha_{k}}{\prod_{j=1}^{K} \prod_{k=1}^{K}\left(z_{k}-\alpha_{j}\right)\left(z_{k}+\alpha_{j}\right)} \\
& \quad \times d z_{1} \ldots d z_{K} .
\end{aligned}
$$


Using this lemma, we can reformulate Theorem 4.2 and Conjecture 5.2 as

$$
\begin{aligned}
& \int_{U S p(2 N)} \frac{\prod_{k=1}^{K} \Lambda_{A}\left(\mathrm{e}^{-\alpha_{k}}\right)}{\prod_{q=1}^{Q} \Lambda_{A}\left(\mathrm{e}^{-\gamma_{q}}\right)} d A \\
& =\mathrm{e}^{-N / 2 \sum_{k=1}^{K} \alpha_{k}} \frac{(-1)^{K(K-1) / 2} 2^{K}}{K !(2 \pi i)^{K}} \\
& \quad \times \int_{\left|z_{i}\right|=1} \frac{h_{S}\left(z_{1}, \ldots, z_{K} ; \gamma\right) \Delta\left(z_{1}^{2}, \ldots, z_{K}^{2}\right)^{2} \prod_{k=1}^{K} z_{k}}{\prod_{j=1}^{K} \prod_{k=1}^{K}\left(z_{k}-\alpha_{j}\right)\left(z_{k}+\alpha_{j}\right)} \\
& \quad \times d z_{1} \ldots d z_{K},
\end{aligned}
$$

and

$$
\begin{aligned}
& \sum_{0<d \leq X} \frac{\prod_{k=1}^{K} L\left(1 / 2+\alpha_{k}, \chi_{d}\right)}{\prod_{q=1}^{Q} L\left(1 / 2+\gamma_{q}, \chi_{d}\right)} \\
& =\sum_{0<d \leq X}\left(\frac{|d|}{\pi}\right)^{-1 / 2 \sum_{k=1}^{K} \alpha_{k}} \frac{(-1)^{K(K-1) / 2} 2^{K}}{K !(2 \pi i)^{K}} \\
& \quad \times \int_{\left|z_{i}\right|=1} \frac{H_{\mathcal{D}+, d, \alpha, \gamma}\left(z_{1}, \ldots, z_{K} ; \gamma\right) \Delta\left(z_{1}^{2}, \ldots, z_{K}^{2}\right)^{2} \prod_{k=1}^{K} z_{k}}{\prod_{j=1}^{K} \prod_{k=1}^{K}\left(z_{k}-\alpha_{j}\right)\left(z_{k}+\alpha_{j}\right)}
\end{aligned}
$$

$$
\times d z_{1} \cdots d z_{K}+O\left(X^{1 / 2+\epsilon}\right)
$$

There is a similar reformulation of Theorems 4.3 and 4.4 and Conjectures 5.3 and 5.4 .

\section{Examples and Applications}

\subsection{Farmer's conjecture revisited}

We first give a more precise version of Farmer's "First Ratios Conjecture", formula (1.1). We use Conjecture 5.1 with $K=L=1$. In this case, $\Xi_{1,1}=$ $\{(1),(12)\}$ consists of the identity permutation and the transposition (12). 
We identify $\alpha_{1}=\alpha, \alpha_{2}=-\beta, \gamma_{1}=\gamma$ and $\delta_{1}=\delta$. Then Conjecture 5.1 tells us that

$$
\begin{aligned}
\int_{0}^{T} & \frac{\zeta(s+\alpha) \zeta(1-s+\beta)}{\zeta(s+\gamma) \zeta(1-s+\delta)} d t \\
= & \int_{0}^{T}\left(Y_{U} A_{\zeta}(\alpha, \beta ; \gamma ; \delta)+\left(\frac{t}{2 \pi}\right)^{-\alpha-\beta} Y_{U} A_{\zeta}(-\beta,-\alpha ; \gamma ; \delta)\right) \\
& \quad \times d t+O\left(T^{1 / 2+\epsilon}\right) .
\end{aligned}
$$

We see that

$$
Y_{U}(\alpha ; \beta ; \gamma ; \delta)=\frac{\zeta(1+\alpha+\beta) \zeta(1+\gamma+\delta)}{\zeta(1+\alpha+\delta) \zeta(1+\beta+\gamma)}
$$

and

$$
\begin{aligned}
A_{\zeta}(\alpha ; \beta ; \gamma ; \delta)= & \prod_{p} \frac{\left(1-1 / p^{1+\alpha+\beta}\right)\left(1-1 / p^{1+\gamma+\delta}\right)}{\left(1-1 / p^{1+\alpha+\delta}\right)\left(1-1 / p^{1+\beta+\gamma)}\right.} \\
& \times \int_{0}^{1} \frac{\left(1-e(\theta) / p^{1 / 2+\gamma}\right)\left(1-e(-\theta) / p^{1 / 2+\delta}\right)}{\left(1-e(\theta) / p^{1 / 2+\alpha}\right)\left(1-e(-\theta) / p^{1 / 2+\beta}\right)} d \theta
\end{aligned}
$$

For values of $\alpha, \beta, \gamma, \delta \rightarrow 0$ we have asymptotically

$$
Y_{U}(\alpha ; \beta ; \gamma ; \delta) \sim \frac{(\alpha+\delta)(\beta+\gamma)}{(\alpha+\beta)(\gamma+\delta)},
$$

$A_{\zeta} \sim 1$, and $\frac{t}{2 \pi}$ can be replaced by $T$; to a first order approximation, we then have

$$
\begin{aligned}
& \frac{1}{T} \int_{0}^{T} \frac{\zeta(s+\alpha) \zeta(1-s+\beta)}{\zeta(s+\gamma) \zeta(1-s+\delta)} d t \\
& \quad \sim \frac{(\alpha+\delta)(\beta+\gamma)}{(\alpha+\beta)(\gamma+\delta)}+T^{-\alpha-\beta} \frac{(-\beta+\delta)(-\alpha+\gamma)}{(-\beta-\alpha)(\gamma+\delta)} \\
& \quad=1+\left(1-T^{-\alpha-\beta}\right) \frac{(\alpha-\gamma)(\beta-\delta)}{(\alpha+\beta)(\gamma+\delta)}
\end{aligned}
$$

which recovers Farmer's original conjecture. 


\subsection{Logarithmic derivatives of $\zeta(s)$}

Goldston, Gonek, and Montgomery [18] proved, assuming the Riemann hypothesis, that

$$
\begin{aligned}
\frac{1}{T} \int_{0}^{T}\left|\frac{\zeta^{\prime}(1 / 2+r+i t)}{\zeta(1 / 2+r+i t)}\right|^{2} d t & \sim \sum_{p} \frac{\log ^{2} p}{p^{1+2 r}-1}-\frac{T^{-2 r}}{4 r^{2}} \\
& +\log ^{2} T \int_{1}^{\infty}(F(\alpha, T)-1) T^{-2 r \alpha} d \alpha
\end{aligned}
$$

uniformly for $T^{-1 / 2} \log T \ll r \ll 1$, where $F(\alpha, T)$ is Montgomery's pair correlation function. Montgomery's function is expected to satisfy $F(\alpha, T)=$ $1+o(1)$ uniformly for bounded $\alpha$ so that the term involving $F$ is expected to be small. Also, the sum over primes is $\sim \frac{1}{4 r^{2}}$ as $r \rightarrow 0$.

Here we obtain a conjecture for this quantity which is more precise than the GGM formula, in that it contains some lower order terms and we expect it to be accurate with a square-root error term. We deduce our conjecture by differentiating the formula of the last section with respect to $\alpha$ and $\beta$ and setting $\alpha=\beta=\gamma=\delta=r$. To help compute this, the following formulas, about a function $f$ which is analytic in a neighborhood of the origin, are helpful:

$$
\left.\frac{d}{d a} \frac{f(a+b) f(c+d)}{f(a+d) f(b+c)}\right|_{a=b=c=d=r}=\left.\frac{d}{d b} \frac{f(a+b) f(c+d)}{f(a+d) f(b+c)}\right|_{a=b=c=d=r}=0
$$

and

$$
\begin{aligned}
& \left.\frac{d}{d a} \frac{d}{d b} \frac{f(a+b) f(c+d)}{f(a+d) f(b+c)}\right|_{a=b=c=d=r} \\
& \quad=\frac{f^{\prime \prime}(2 r)}{f(2 r)}-\left(\frac{f^{\prime}(2 r)}{f(2 r)}\right)^{2}=\left.\frac{d}{d x} \frac{f^{\prime}(x)}{f(x)}\right|_{x=2 r}=\left.\frac{d^{2}}{d x^{2}} \log (f(x))\right|_{x=2 r}
\end{aligned}
$$

Also,

$$
\left.\frac{d}{d a} \frac{d}{d b} \frac{f(a) f(b)}{f(c) f(d)}\right|_{a=b=c=d=r}=\left(\frac{f^{\prime}}{f}(2 r)\right)^{2}
$$


Thus, we can now calculate

$$
\left.\frac{d}{d \alpha} \frac{d}{d \beta} Y_{U} A_{\zeta}(\alpha ; \beta ; \gamma ; \delta)\right|_{\alpha=\beta=\gamma=\delta=r}
$$

$$
=\left(\frac{\zeta^{\prime}}{\zeta}\right)^{\prime}(1+2 r)+\sum_{p}\left(\frac{-p^{1+2 r} \log ^{2} p}{\left(p^{1+2 r}-1\right)^{2}}+\int_{0}^{1} \frac{\log ^{2} p}{\left(e(\theta) p^{1 / 2+r}-1\right)^{2}} d \theta\right),
$$

and

$$
\begin{gathered}
\left.\frac{d}{d \alpha} \frac{d}{d \beta}\left(\frac{t}{2 \pi}\right)^{-\alpha-\beta} Y_{U} A_{\zeta}(-\beta ;-\alpha ; \gamma ; \delta)\right|_{\alpha=\beta=\gamma=\delta=r} \\
=\left(\frac{t}{2 \pi}\right)^{-2 r} A_{\zeta}(-r,-r, r, r) \zeta(1-2 r) \zeta(1+2 r)
\end{gathered}
$$

Thus, we have

\section{Conjecture 7.1.}

$$
\begin{aligned}
\frac{1}{T} \int_{0}^{T} & \left|\frac{\zeta^{\prime}(1 / 2+r+i t)}{\zeta(1 / 2+r+i t)}\right|^{2} d t \\
= & \left(\frac{\zeta^{\prime}}{\zeta}\right)^{\prime}(1+2 r)+\left(\frac{T}{2 \pi}\right)^{-2 r} A_{\zeta}(-r,-r, r, r) \frac{\zeta(1-2 r) \zeta(1+2 r)}{1-2 r} \\
& +c(r)+O\left(T^{-1 / 2+\epsilon}\right),
\end{aligned}
$$

where $c(r)$ is a function of $r$ which is uniformly bounded for $|r|<1 / 4-\epsilon$ and is given by

$$
c(r)=\sum_{p}\left(\frac{-p^{1+2 r} \log ^{2} p}{\left(p^{1+2 r}-1\right)^{2}}+\int_{0}^{1} \frac{\log ^{2} p}{\left(e(\theta) p^{1 / 2+r}-1\right)^{2}} d \theta\right) .
$$

\subsection{A conjecture of Keating and Snaith}

A conjecture of Keating and Snaith is

$$
\frac{1}{T} \int_{0}^{T} \frac{\zeta(1 / 2+i t)^{K}}{\zeta(1 / 2-i t)^{K}} d t \sim G(1-K) G(1+K) b_{K}(\log T)^{-K^{2}}
$$


where $G$ is Barnes double Gamma-function and

$$
b_{K}=\prod_{p}\left(1-\frac{1}{p}\right)^{-K^{2}} \sum_{j=0}^{\infty} \frac{\Gamma(1+K) \Gamma(1-K)}{\Gamma(1+K-j) \Gamma(1-K-j) j^{!^{2} p^{j}}} .
$$

Note that if $K$ is a positive integer, then $b_{K}=0$. Here we consider the case that $K$ is a positive integer, but integrate a ratio of shifted zetafunctions. For $\alpha_{k}$ and $\delta_{k}$ with positive real parts we have, as a consequence of Conjecture 5.1,

$$
\frac{1}{T} \int_{0}^{T} \prod_{k=1}^{K} \frac{\zeta\left(s+\alpha_{k}\right)}{\zeta\left(1-s+\delta_{k}\right)} d t=B(\alpha, \delta) \prod_{i, j=1}^{K} \zeta\left(1+\alpha_{i}+\delta_{j}\right)^{-1}+O\left(T^{1 / 2+\epsilon}\right)
$$

where

$$
B(\alpha, \delta)=\prod_{p} \prod_{i, j=1}^{K}\left(1-\frac{1}{p^{1+\alpha_{i}+\delta_{j}}}\right)^{-1} \sum_{\sum a_{i}=\sum d_{i}} \frac{\prod \mu\left(p^{d_{j}}\right)}{p^{\sum a_{i}\left(1 / 2+\alpha_{i}\right)+\sum d_{i}\left(1 / 2+\delta_{i}\right)}} .
$$

Note that the product over primes in $B$ is absolutely convergent for sufficiently small values of the shifts $\alpha_{i}, \delta_{i}$ and is equal to the $b_{K}$ in Keating and Snaith's formula when all of the shifts are 0. Also, the size of this expression is about $(\log T)^{-K^{2}}$ when all of the shifts have order of magnitude $1 / \log T$.

\subsection{Discrete moments of $\zeta$}

Let $\rho=\beta+i \gamma$ stand for a typical complex zero of the Riemann zeta-function. The Riemann hypothesis, which we assume here, asserts that all $\beta=1 / 2$. The number of $\gamma \leq T$ was proven by Riemann and von Mangoldt to equal $\frac{T}{2 \pi} \log \frac{T}{2 \pi e}+O(\log T)$. Chris Hughes [22-24] has conjectured a formula for the leading term of

$$
\sum_{\gamma \leq T}\left|\zeta^{\prime}(\rho)\right|^{s}
$$

for complex $s$ with $\sigma>-3$. His conjecture is based on an exact formula he proved for the analogous random matrix moment:

$$
\int_{U(N)} \sum_{n=1}^{N}\left|\Lambda_{A}^{\prime}\left(\mathrm{e}^{i \theta_{n}}\right)\right|^{s} d A_{N}=\frac{G^{2}(s / 2+2) G(N+s+2) G(N)}{G(s+3) G^{2}(N+s / 2+1)}
$$


for $\Re s>-3$. Here the $\mathrm{e}^{i \theta_{n}}$ are the zeros of the characteristic polynomial $\Lambda_{A}$ and $G$ is the Barnes double Gamma-function. It should be possible to use our ratios conjecture to determine the lower order terms of the discrete moments $\sum_{\gamma \leq T}\left|\zeta^{\prime}(\rho)\right|^{2 k}$ for positive integer $k$. Conrey and Snaith [10] consider these moments in the cases $k=2$ and 4 .

Now, we compute a conjecture for

$$
D(a, c)=\sum_{\gamma \leq T} \frac{\zeta(\rho+a)}{\zeta(\rho+c)}
$$

where $\Re a, \Re c>0$, a sum which was considered in Farmer's paper $[9,14]$. Farmer's conjecture for this sum is

$$
D(a, c) \sim \frac{T}{2 \pi}\left(\log T+\left(1-T^{-a}\right)\left(\frac{1}{c}-\frac{1}{a}\right)\right) .
$$

We now give a more precise conjecture based on our ratios conjecture.

By Cauchy's formula,

$$
D(a, c)=\frac{1}{2 \pi i} \int_{\mathcal{C}} \frac{\zeta^{\prime}}{\zeta}(s) \frac{\zeta(s+a)}{\zeta(s+c)} d s
$$

where $\mathcal{C}$ is a tall, thin rectangular path (with vertices $1 / 2 \pm \alpha, 1 / 2 \pm \alpha+i T$, which encloses the zeros $1 / 2+i \gamma$ for $0<\gamma<T$. Here $\alpha>0$ is smaller than the real parts of $a$ and $b$. The parameter $T$ can be slightly adjusted if necessary to conclude that the integrals on the horizontal portions of the path are $\ll T^{\epsilon}$. Also, the integral on the right hand path $1 / 2+\alpha+i t, 0 \leq$ $t \leq T$ is $\ll T^{\epsilon}$, as can be seen by moving the path of integration to the right of $\sigma=1$ and integrating term-by-term. Thus, we have

$$
D(a, c)=\frac{-1}{2 \pi} \int_{0}^{T} \frac{\zeta^{\prime}}{\zeta}\left(\frac{1}{2}-\alpha+i t\right) \frac{\zeta(1 / 2-\alpha+a+i t)}{\zeta(1 / 2-\alpha+c+i t)} d t+O\left(T^{\epsilon}\right)
$$

Now we use the functional equation

$$
\frac{\zeta^{\prime}}{\zeta}(s)=\frac{\chi^{\prime}}{\chi}(s)-\frac{\zeta^{\prime}}{\zeta}(1-s)
$$

and split the integral into two pieces. The part with the $\chi^{\prime} / \chi$ can be treated much as the first integral (on the $1 / 2+\alpha$-line) by moving the path of integration to the right and into the region of absolute convergence of the Dirichlet series where we can integrate term-by-term. Note that this Dirichlet series 
begins with a 1 , whereas in the first integral the series had no constant term. Thus, the contribution from this integral is

$$
\begin{aligned}
& =\frac{-1}{2 \pi} \int_{0}^{T} \frac{\chi^{\prime}}{\chi}(2+i t) d t+O\left(T^{\epsilon}\right) \\
& =\frac{1}{2 \pi} \int_{0}^{T} \log \frac{t}{2 \pi} d t+O\left(T^{\epsilon}\right)
\end{aligned}
$$

Thus, we have

$$
\begin{aligned}
D(a, b)= & \frac{1}{2 \pi} \int_{0}^{T}\left(\log \frac{t}{2 \pi}+\frac{\zeta^{\prime}}{\zeta}(1 / 2+\alpha-i t) \frac{\zeta(1 / 2-\alpha+a+i t)}{\zeta(1 / 2-\alpha+c+i t)}\right) d t \\
& +O\left(T^{\epsilon}\right) \\
= & \left.\frac{1}{2 \pi} \int_{0}^{T}\left(\log \frac{t}{2 \pi}+\frac{d}{d \beta} \frac{\zeta(1-s+\alpha+\beta) \zeta(s-\alpha+a)}{\zeta(1-s+\alpha) \zeta(s-\alpha+c)}\right) d t\right|_{\beta=0} \\
& +O\left(T^{\epsilon}\right),
\end{aligned}
$$

where $s=1 / 2+i t$. By (7.1), we have

$$
\begin{aligned}
\int_{0}^{T} & \frac{\zeta(1-s+\alpha+\beta) \zeta(s-\alpha+a)}{\zeta(1-s+\alpha) \zeta(s-\alpha+c)} d t \\
= & O\left(T^{1 / 2+\epsilon}\right)+\int_{0}^{T}\left(Y_{U} A_{\zeta}(a-\alpha, \alpha+\beta ; c-\alpha, \alpha)\right. \\
& \left.\quad+\left(\frac{t}{2 \pi}\right)^{-a-\beta} Y_{U} A_{\zeta}(-\alpha-\beta, \alpha-a ; c-\alpha, \alpha)\right) d t
\end{aligned}
$$

Now it can be easily calculated that

$$
Y_{U}(x, y ; z, w)=\frac{\zeta(1+x+y) \zeta(1+z+w)}{\zeta(1+x+w) \zeta(1+y+z)}
$$

and

$$
A_{\zeta}(x, y ; z, w)=\prod_{p} \frac{\left(1-1 / p^{1+z+w}\right)\left(1-1 / p^{1+y+z}-1 / p^{1+x+w}+1 / p^{1+z+w}\right)}{\left(1-1 / p^{1+y+z}\right)\left(1-1 / p^{1+x+w}\right)}
$$


Thus,

$$
\begin{aligned}
Y_{U} & A_{\zeta}(a-\alpha, \alpha+\beta ; c-\alpha, \alpha) \\
= & \frac{\zeta(1+a+\beta) \zeta(1+c)}{\zeta(1+a) \zeta(1+c+\beta)} \\
& \times \prod_{p} \frac{\left(1-1 / p^{1+c}\right)\left(1-1 / p^{1+\beta+c}-1 / p^{1+a}+1 / p^{1+c}\right)}{\left(1-1 / p^{1+\beta+c}\right)\left(1-1 / p^{1+a}\right)}
\end{aligned}
$$

and

$$
\begin{aligned}
Y_{U} A_{\zeta}(-\alpha-\beta, \alpha-a ; c-\alpha, \alpha) \\
=\frac{\zeta(1-a-\beta) \zeta(1+c)}{\zeta(1-\beta) \zeta(1+c-a)} \\
\quad \times \prod_{p} \frac{\left(1-1 / p^{1+c}\right)\left(1-1 / p^{1+c-a}-1 / p^{1-\beta}+1 / p^{1+c}\right)}{\left(1-1 / p^{1+c-a}\right)\left(1-1 / p^{1-\beta}\right)} .
\end{aligned}
$$

If we differentiate the first expression with respect to $\beta$ and set $\beta=0$, we get

$$
\frac{\zeta^{\prime}}{\zeta}(1+a)-\frac{\zeta^{\prime}}{\zeta}(1+c)-\sum_{p} \frac{\log p}{p^{2+a+c}}\left(1-\frac{1}{p^{1+c}}-\frac{1}{p^{1+a}}\right)^{-1}\left(1-\frac{1}{p^{1+c}}\right)^{-1} .
$$

The second expression gets multiplied by $\left(\frac{t}{2 \pi}\right)^{-a-\beta}$; then we differentiate with respect to $\beta$ and set $\beta=0$. Note that because of the factor $\zeta(1-\beta)$ in the denominator we only have to differentiate that term since it gives 0 when $\beta=0$. Thus, the second term results in a contribution of

$-\left(\frac{t}{2 \pi}\right)^{-a} \frac{\zeta(1-a) \zeta(1+c)}{\zeta(1+c-a)} \prod_{p} \frac{\left(1-1 / p^{1+c}\right)\left(1-1 / p^{1+c-a}-1 / p+1 / p^{1+c}\right)}{\left(1-1 / p^{1+c-a}\right)(1-1 / p)}$.

Thus, we have 
Conjecture 7.2. Let $a$ and $c$ have non-negative real parts and satisfy $|a|,|c| \gg(\log T)^{-1}$. Then

$$
\begin{aligned}
\frac{1}{T} \sum_{\gamma<T} \frac{\zeta(\rho+a)}{\zeta(\rho+c)}= & \frac{1}{2 \pi} \int_{0}^{T}\left(\log \frac{t}{2 \pi}+\frac{\zeta^{\prime}}{\zeta}(1+a)-\frac{\zeta^{\prime}}{\zeta}(1+c)\right. \\
& -\sum_{p} \frac{\log p}{p^{2+a+c}}\left(1-\frac{1}{p^{1+c}}-\frac{1}{p^{1+a}}\right)^{-1}\left(1-\frac{1}{p^{1+c}}\right)^{-1} \\
& -\left(\frac{t}{2 \pi}\right)^{-a} \frac{\zeta(1-a) \zeta(1+c)}{\zeta(1+c-a)} \\
& \left.\times \prod_{p} \frac{\left(1-1 / p^{1+c}\right)\left(1-1 / p^{1+c-a}-1 / p+1 / p^{1+c}\right)}{\left(1-1 / p^{1+c-a}\right)(1-1 / p)}\right) d t \\
& +O\left(T^{1 / 2+\epsilon}\right) .
\end{aligned}
$$

This agrees with Farmer's conjecture (7.21).

More applications of the ratios conjecture are given by Conrey and Snaith [10].

\section{References}

[1] J. Baik, P. Deift and E. Strahov, Products and ratios of characteristic polynomials of random Hermitian matrices. Integrability, topological solitons and beyond, J. Math. Phys. 44(8) (2003), 3657-3670, arXiv:math-ph/0304016.

[2] E.L. Basor and P.J. Forrester, Formulas for the evaluation of Toeplitz determinants with rational generating functions, Math. Nachr. 170 (1994), 5-18.

[3] D. Bump and A. Gamburd, On the averages of characteristic polynomials from classical groups (English summary), Comm. Math. Phys. 265(1) (2006), 227-274.

[4] J.B. Conrey and D.W. Farmer, Mean values of L-functions and symmetry, Internat. Math. Res. Notices 17 (2000), 883-908.

[5] J.B. Conrey, D.W. Farmer, J.P. Keating, M.O. Rubinstein and N.C. Snaith, Autocorrelation of random matrix polynomials, Comm. Math. Phys. 237(3) (2003), 365-395. 
[6] - Integral moments of L-functions, Proc. London Math. Soc. (3) 91(1) (2005), 33-104, arXiv math.NT/0206018.

[7] J.B. Conrey, P. Forrester and N.C. Snaith, Averages of ratios of characteristic polynomials for the classical compact groups, Int. Math. Res. Not. (7) (2005), 397-431.

[8] J.B. Conrey, D.W. Farmer and M.R. Zirnbauer, Howe pairs, supersymmetry, and ratios of random characteristic polynomials for the unitary groups $U_{N}$, preprint, arXiv:math-ph/0511024.

[9] J.B. Conrey, A. Ghosh and S.M. Gonek, Simple zeros of the Riemann zeta-function, Proc. London Math. Soc. (3) 76(3) (1998), 497-522.

[10] J.B. Conrey and N.C. Snaith, Applications of the L-functions ratios conjecture, Proc. Lond. Math. Soc. (3) 94(3) (2007), 594-646.

[11] H. Davenport, Multiplicative number theory, Third edition, Revised and with a preface by Hugh L. Montgomery, Graduate Texts in Mathematics, 74, Springer-Verlag, New York, 2000.

[12] K.M. Day, Toeplitz matrices generated by the Laurent series expansion of an arbitrary rational function, Trans. Amer. Math. Soc. 206 (1975), 224-245.

[13] A. Diaconu, D. Goldfeld and J. Hoffstein, Multiple Dirichlet series and moments of zeta- and L-functions, Compos. Math. 139(3) (2003), 297-360, ArXiv:math.nt/0110092.

[14] D.W. Farmer, Long mollifiers of the Riemann zeta-function, Mathematika 40(1) (1993), 71-87.

[15] - Mean values of $\zeta^{\prime} / \zeta$ and the Gaussian unitary ensemble hypothesis, Internat. Math. Res. Notices (2) (1995), 71-82.

[16] - Modeling families of L-functions, in 'Ranks of Elliptic Curves and Random Matrix Theory,' London Math. Soc. Lecture Note Ser., 341, Cambridge University Press, Cambridge, 2007, 53-69, arXiv:math.NT/0511107.

[17] Y.V. Fyodorov and E. Strahov, An exact formula for general spectral correlation function of random Hermitian matrices, J. Phys. A Math. Gen. 36(12) (2003), 3203-3213, arXiv:math-ph/0204051.

[18] D.A. Goldston, S.M. Gonek and H.L. Montgomery, Mean values of the logarithmic derivative of the Riemann zeta-function with applications to primes in short intervals, J. Reine Angew. Math. 537 (2001), 105-126. 
[19] D.A. Goldston and H.L. Montgomery, Pair correlation of zeros and primes in short intervals, in 'Analytic Number Theory and Diophantine Problems (Stillwater, OK, 1984)', Progr. Math., 70, Birkhäuser, Boston, MA, 1987, 183-203.

[20] D.A. Hejhal, On the triple correlation of zeros of the zeta function, Internat. Math. Res. Notices (7) (1994), $293 \mathrm{ff}$.

[21] A. Huckleberry, A. Püttmann and M.R. Zirnbauer, Haar expectations of ratios of random characteristic polynomials, preprint, arXiv:0709:1215.

[22] C.P. Hughes, J.P. Keating and N. O'Connell, Random matrix theory and the derivative of the Riemann zeta function, R. Soc. Lond. Proc. Ser. A Math. Phys. Eng. Sci. 456(2003) (2000), 2611-2627.

[23] C.P. Hughes, On the characteristic polynomial of a random unitary matrix and the Riemann zeta function, $\mathrm{PhD}$ thesis, http://www.tau.ac.il/ hughes/papers.html.

[24] C.P. Hughes, J.P. Keating and N. O'Connell, Random matrix theory and the derivative of the Riemann zeta function, R. Soc. Lond. Proc. Ser. A Math. Phys. Eng. Sci. 456(2003) (2000), 2611-2627.

[25] H. Iwaniec, Topics in classical automorphic forms, Graduate Studies in Mathematics, 17, American Mathematical Society, Providence, RI, 1997.

[26] N.M. Katz and P. Sarnak, Random matrices, Frobenius eigenvalues, and monodromy, American Mathematical Society Colloquium Publications, 45, American Mathematical Society, Providence, RI, 1999.

[27] J.P. Keating and N.C. Snaith, Random matrix theory and $\zeta(1 / 2+i t)$, Comm. Math. Phys. 214(1) (2000), 57-89.

[28] - Random matrix theory and L-functions at $s=1 / 2$, Comm. Math. Phys. 214(1) (2000), 91-110.

[29] S.J. Miller, A symplectic test of the L-functions ratios conjecture, Int. Math. Res. Not. IMRN (3) (2008), Art. ID rnm146, 36pp, arXiv/0704.0927.

[30] H.L. Montgomery, The pair correlation of zeros of the zeta function, in 'Analytic Number Theory (Proc. Sympos. Pure Math., XXIV, St Louis University, St Louis, MO, 1972)', American Mathematical Society, Providence, RI, 1973, 181-193. 
636 Brian Conrey, David W. Farmer and Martin R. Zirnbauer

[31] Z. Rudnick and P. Sarnak, Zeros of principal L-functions and random matrix theory. A celebration of John F. Nash, Jr, Duke Math. J. 81(2) (1996), 269-322.

[32] E.C. Titchmarsh, The theory of the Riemann zeta-function, Second edition, Edited and with a preface by D. R. Heath-Brown, The Clarendon Press, Oxford University Press, Oxford, 1986.

American Institute of Mathematics

360 Portage Avenue

Palo Alto

CA 94306-2244

USA

E-mail address: conrey@aimath.org

E-mail address: farmer@aimath.org

Institut Fuer Theoretische Physik

UNIVERSITAT ZU KOELN

ZulPICHER STRASSE 77

D-50937 KOELN

GERMANY

E-mail address: zirn@thp.uni-koeln.de

Received December 13, 2007 